\title{
Coset conformal field theory and instanton counting on $\mathbb{C}^{2} / \mathbb{Z}_{p}$
}

\author{
M.N. Alfimov, ${ }^{a, b, c, d}$ A.A. Belavin ${ }^{d, e, f}$ and G.M. Tarnopolsky ${ }^{f, g}$ \\ ${ }^{a}$ LPT, Ecole Normale Superieure, \\ 24, rue Lhomond 75005 Paris, France \\ ${ }^{b}$ Institut de Physique Théorique, Orme des Merisiers, CEA Saclay, \\ 91191 Gif-sur-Yvette Cedex, France \\ ${ }^{c}$ P.N. Lebedev Physical Institute, \\ Leninskiy pr. 53, 119991 Moscow, Russia \\ ${ }^{d}$ Moscow Institute of Physics and Technology, \\ Institutskiy per. 9, 141700 Dolgoprudny, Russia \\ e Institute for Information Transmission Problems, \\ Bolshoi Karetnyi per. 19, 127994, Moscow, Russia \\ ${ }^{f}$ Landau Institute for Theoretical Physics, \\ Akademika Semenova pr. 1A, 142432 Chernogolovka, Russia \\ ${ }^{g}$ Department of Physics, Princeton University, \\ Jadwin Hall, Washington Road, Princeton, NJ, 08540, U.S.A. \\ E-mail: alfimov@lpt.ens.fr, sashabelavin@gmail.com, \\ grigory@princeton.edu
}

ABSTRACT: We study conformal field theory with the symmetry algebra $\mathcal{A}(2, p)=$ $\widehat{\mathfrak{g l}}(n)_{2} / \widehat{\mathfrak{g l}}(n-p)_{2}$. In order to support the conjecture that this algebra acts on the moduli space of instantons on $\mathbb{C}^{2} / \mathbb{Z}_{p}$, we calculate the characters of its representations and check their coincidence with the generating functions of the fixed points of the moduli space of instantons.

We show that the algebra $\mathcal{A}(2, p)$ can be realized in two ways. The first realization is connected with the cross-product of $p$ Virasoro and $p$ Heisenberg algebras: $\mathcal{H}^{p} \times \operatorname{Vir}^{p}$. The second realization is connected with: $\mathcal{H}^{p} \times \widehat{\mathfrak{s l}}(p)_{2} \times\left(\widehat{\mathfrak{s l}}(2)_{p} \times \widehat{\mathfrak{s l}}(2)_{n-p} / \widehat{\mathfrak{s l}}(2)_{n}\right)$. The equivalence of these two realizations provides the non-trivial identity for the characters of $\mathcal{A}(2, p)$.

The moduli space of instantons on $\mathbb{C}^{2} / \mathbb{Z}_{p}$ admits two different compactifications. This leads to two different bases for the representations of $\mathcal{A}(2, p)$. We use this fact to explain the existence of two forms of the instanton pure partition functions.

KEYWords: Supersymmetric gauge theory, Conformal and W Symmetry

ARXIV EPRINT: 1306.3938 


\section{Contents}

1 Introduction 1

2 Counting of the torus fixed points on the moduli space of instantons $\quad 3$

2.1 Fixed points on the moduli space of $\mathrm{U}(2)$ instantons on $\mathbb{C}^{2} / \mathbb{Z}_{p} \quad 3$

2.2 Counting of the non equivalent generating functions of the colored Young diagrams

$\begin{array}{lll}3 & \text { First realization of the algebra } \mathcal{A}(2, p) & 7\end{array}$

3.1 The $p$ models with Virasoro symmetry 8

3.2 Comparison with the generating functions of the colored Young diagrams 12

4 Second realization of the algebra $\mathcal{A}(2, p) \quad 13$

4.1 Representations of the coset $\widehat{\mathfrak{s l}}(2)_{p} \times \widehat{\mathfrak{s l}}(2)_{n-p} / \widehat{\mathfrak{s l}}(2)_{n} \quad 14$

$\begin{array}{ll}4.2 \text { Product of consecutive Minimal models } & 15\end{array}$

$\begin{array}{ll}\text { 4.3 Comparison with the first realization of } \mathcal{A}(2, p) & 16\end{array}$

5 Comparison of the instanton partition functions 18

$\begin{array}{llr}5.1 \text { First compactification } & 18\end{array}$

$\begin{array}{ll}5.2 \text { Second compactification } & 19\end{array}$

5.3 Bases in conformal field theories and the equality of instanton partition functions 20

$\begin{array}{ll}\text { A Symmetries of the generating functions } & 21\end{array}$

B Conformal field theories based on the coset 23

\section{Introduction}

In recent years the remarkable relation between two dimensional conformal field theories and four dimensional $\mathcal{N}=2$ supersymmetric Yang-Mills theories experienced a considerable development. The original so-called AGT relation proposed in [1] states the equality between the correlation functions in the Liouville field theory and the partition function of the $\mathcal{N}=2$ supersymmetric Yang-Mills theory with the $\mathrm{SU}(2)$ gauge group (for the generalizations of the AGT correspondence for the other gauge groups and conformal field theories see [2-12]). The partition function of the $\mathcal{N}=2$ supersymmetric gauge theory can be calculated as the integral over the moduli space of instantons $\mathcal{M}$. With the proper regularization, this integral was computed explicitly [13]. This was achieved using the localization theorem, which shows that the integral is fully determined by the fixed points of some abelian group (torus) acting on the moduli space $\mathcal{M}[14]$. 
In the present work we consider the $\mathrm{U}(r)$ instantons on $\mathbb{C}^{2} / \mathbb{Z}_{p}$ - the solutions of the self-duality equation, with the additional condition for the gauge field:

$$
A_{\mu}\left(z_{1}, z_{2}\right)=A_{\mu}\left(\omega z_{1}, \omega^{-1} z_{2}\right), \quad \omega^{p}=1 .
$$

The nontrivial fact about the moduli space of instantons $\mathcal{M}$ is that one can construct the action of some symmetry algebra $\mathcal{A}$ on the equivariant cohomologies of the moduli space $\mathcal{M}$. The first examples of such action were given by Nakajima in $[15,16]$ for the cases of Heisenberg and Kac-Moody algebras. In [17] it was shown that the basis in the space of the equivariant cohomologies can be labelled by the fixed points of the torus acting on the moduli space. Therefore it is natural to assume the existence of the special basis of the geometrical origin in the representation of $\mathcal{A}$, elements of which being in one to one correspondence with the torus fixed points on the moduli space. This basis has a number of remarkable properties, which are listed in [18].

In the work [8] it was suggested that the instanton moduli space of the $\mathcal{N}=2$ supersymmetric $\mathrm{U}(r)$ gauge theory on $\mathbb{C}^{2} / \mathbb{Z}_{p}$ is connected to the algebra $\mathcal{A}(r, p)$, which is realized by the coset

$$
\mathcal{A}(r, p) \stackrel{\text { def }}{=} \frac{\widehat{\mathfrak{g l}}(n)_{r}}{\widehat{\mathfrak{g} l}(n-p)_{r}},
$$

where $n$ is related to the equivariant parameters (to learn more details about this correspondence see [18]). In other words there exists the special basis in the representation of $\mathcal{A}(r, p)$, whose elements are in one to one correspondence with the fixed points of the torus acting on the instanton moduli space $\mathcal{M}$. On the other hand these fixed points can be enumerated by the $r$-tuples of the Young diagrams painted in $p$ colors. Therefore we can associate the specific $r$-tuple of colored Young diagrams to each element of this geometrical basis. Such bases were explicitly constructed for $r=2$ and $p=1$ in [19] and for $r=2$ and $p=2$ in [18] and for $r=1,2$ and $p=2$ in [20].

The present work can be considered as the continuation of the line of studies started in $[9-11,18,20]$. The main goal of this work is to find nontrivial evidence in support of the conjectured correspondence between the algebra $\mathcal{A}(2, p)$ and the moduli space of $\mathrm{U}(2)$ instantons on $\mathbb{C}^{2} / \mathbb{Z}_{p}$, which we denote by $\bigsqcup_{N} \mathcal{M}(2, \mathbb{N})^{\mathbb{Z}_{p}}$. Namely, we check the correspondence between the fixed points on the moduli space of $\mathrm{U}(2)$ instantons on $\mathbb{C}^{2} / \mathbb{Z}_{p}$ and the vectors in the representation of the algebra $\mathcal{A}(2, p)$, by comparing the generating functions of the fixed points and the characters of the representations.

Using the level-rank duality the algebra $\mathcal{A}(2, p)$ can be represented in two ways
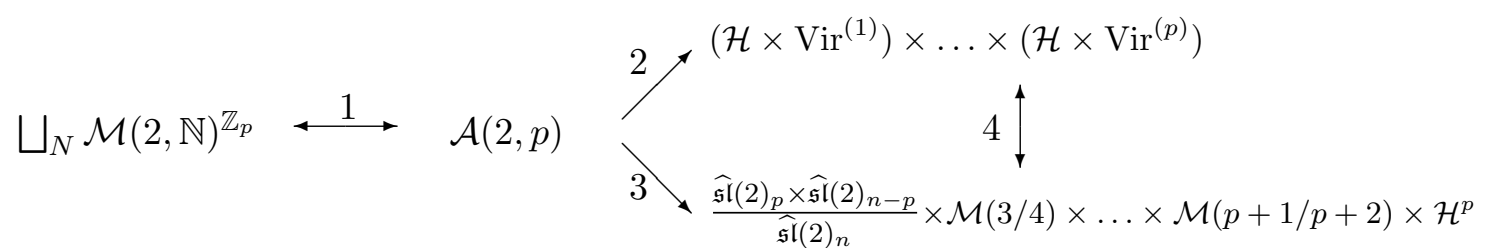
where $\operatorname{Vir}^{(\sigma)}, \sigma=1, \ldots, p$ are the Virasoro algebras with the special central charges $c_{\sigma}, \mathcal{H}$ is the Heisenberg algebra, $\mathcal{M}(m / m+1)$ is the Minimal model $^{1}$ and $\widehat{\mathfrak{s l}}(2)_{p} \times \widehat{\mathfrak{s l}}(2)_{n-p} / \widehat{\mathfrak{s l}}(2)_{n}$ is the coset algebra. In this paper we study the connections (1.3) in detail.

In the section 2 we find the generating function for the fixed points of the moduli space. In the section 3 we study the first realization of $\mathcal{A}(2, p)$ depicted by the arrow 2 on (1.3) and elaborate on the characters of the representation of $\mathcal{A}(2, p)$ in this realization and compare them with the generating functions of the fixed points of the moduli space. In the section 4 we study the second realization of $\mathcal{A}(2, p)$ represented by the arrow 3 on (1.3). Also we find the coincidence of the characters of the first and second realizations of $\mathcal{A}(2, p)$ (arrow 4 on (1.3)). In the section 5 we find the equalities between the instanton partition functions of the $\mathcal{N}=2$ supersymmetric $\mathrm{U}(2)$ pure gauge theory on $\mathbb{C}^{2} / \mathbb{Z}_{p}$ calculated for the different compactifications of the moduli space.

\section{Counting of the torus fixed points on the moduli space of instantons}

In this section we concentrate on the counting of the fixed points of the moduli space. Let us consider the compactification of the moduli space, where the action of the $\mathbb{Z}_{p}$ is lifted to the moduli space of instantons on $\mathbb{C}^{2}$. In this case it is convenient to label the fixed points by the $r$-tuples of Young diagrams with $p$ colors. We are going to introduce the generating functions of such Young diagrams and study the properties of such generating functions.

\subsection{Fixed points on the moduli space of $U(2)$ instantons on $\mathbb{C}^{2} / \mathbb{Z}_{p}$}

Here we confine ourselves to the case of $r=2$, which means that we consider the $\mathrm{U}(2)$ instantons on $\mathbb{C}^{2} / \mathbb{Z}_{p}$, whose moduli space is $\bigsqcup_{N} \mathcal{M}(2, \mathbb{N})^{\mathbb{Z}_{p}}$. It is convenient to numerate the torus fixed points in this case by the pairs of Young diagrams colored in $p$ colors. The coloring goes as follows. We ascribe the color $r$ from 0 to $p-1$ to the corner cell and the colors $r+i-j \bmod p$ to the cell with the coordinates $(i, j)$. For example, the diagram with $r=3$ and $p=4$

\begin{tabular}{|c|c|c|c|c|}
\hline 3 & \begin{tabular}{l|l|}
0 & \\
\end{tabular} & 1 & 2 & \begin{tabular}{l|l}
30 \\
\end{tabular} \\
\hline 2 & \begin{tabular}{l|l}
3 \\
\end{tabular} & 0 & \begin{tabular}{l|l}
1 \\
\end{tabular} & \\
\hline 1 & 2 & \begin{tabular}{l|l}
3 \\
\end{tabular} & 0 & \\
\hline 0 & 1 & & & \\
\hline 3 & & & & \\
\hline
\end{tabular}

In this section we count these fixed points. Because the number of the pairs of Young diagrams colored in $p$ colors is infinite, we need to introduce the grading of these diagrams. One possible way to do that is to count the pairs of Young diagrams with the fixed common size, fixed colors of the corner cells $r_{1}, r_{2}$ and differences $k_{m}$ between the number of cells with the color $m>0$ and the number of cells with the color 0 in both diagrams. We will call the set of numbers $r_{1}, r_{2}$ and $k_{1}, \ldots, k_{p-1}\left(k_{0}=0\right.$ by definition) as the coloring of the Young diagrams. Thus, we determine the generating function of the pair of Young diagrams as follows

$$
\chi_{r_{1}, r_{2}}\left(k_{1}, \ldots, k_{p-1} \mid q\right)=\sum_{\left(Y_{1}, Y_{2}\right) \in \nabla} q^{\frac{\left|Y_{1}\right|+\left|Y_{2}\right|}{p}}
$$

\footnotetext{
${ }^{1}$ Here we have already used another level-rank duality, which gives $\widehat{\mathfrak{s l}}(p)_{2}=\mathcal{M}(3 / 4) \times \ldots \times \mathcal{M}(p+1 / p+2)$.
} 
where

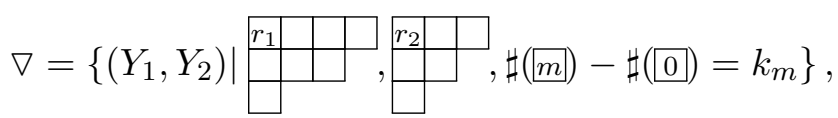

and $|Y|$ is the number of the boxes in the Young diagram $Y$.

It should be noted here that the coloring parameters of the Young diagrams are connected with the topological characteristics of the instantons corresponding to the fixed points of torus action on the moduli space. Let $c_{1}(E)$ be the first Chern class of the gauge bundle and $c_{1}\left(T_{r}\right)$ be the first Chern class of the vector bundle on the ALE space, then

$$
c_{1}(E)=\sum_{r=1}^{p-1} c_{(r)} c_{1}\left(T_{r}\right) .
$$

Denoting the number of Young diagrams with the color of corner cell $r$ by $n_{r}$ we get

$$
c_{(r)}=n_{r}+k_{r+1}-2 k_{r}+k_{r-1}, r=1, \ldots, p-1 .
$$

Utilizing (2.4) one can pass from the description of the generating functions of the Young diagrams in terms of colorings to the equivalent description in terms of Chern classes.

The generating function of the pair of colored Young diagrams can be constructed from the generating function of one colored Young diagram, which can be extracted from [21]. Let $r$ be the color of the corner cell, then for $r=0, \ldots, p-1$ the generating function of one colored Young diagram is defined as

$$
\chi_{r}\left(k_{1}, \ldots, k_{p-1} \mid q\right) \stackrel{\text { def }}{=} \sum_{Y \in \diamond} q^{\frac{|Y|}{p}}
$$

where $\diamond$ is the set of Young diagrams with the particular coloring

$$
\diamond=\left\{Y \mid \begin{array}{|l|l|l}
\hline r & &
\end{array}, \sharp\left((m)-\sharp\left([0)=k_{m}\right\},\right.\right.
$$

where the box with coordinates $(i, j)$ has the color $r+i-j \bmod p$ and $\sharp((m)), \sharp([0)$ - the numbers of the boxes with $m$ and 0 colors respectively. For example we have

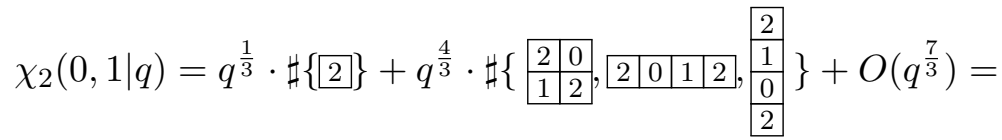

$$
\begin{aligned}
& =q^{\frac{1}{3}}+3 q^{\frac{4}{3}}+O\left(q^{\frac{7}{3}}\right) \text {. }
\end{aligned}
$$

Introducing a convenient notation for the character of the highest weight representation of the Heisenberg algebra ${ }^{2} \mathcal{H}$

$$
\chi_{B}(q)=\prod_{n=1}^{\infty} \frac{1}{1-q^{n}}
$$

\footnotetext{
${ }^{2}$ The Heisenberg algebra consists of the elements $a_{k}$ without $a_{0}$ and has the commutation relations $\left[a_{n}, a_{m}\right]=n \delta_{n+m, 0}$. The highest weight representation of this algebra (Fock module) has the vacuum state $|0\rangle: a_{n}|0\rangle=0$ for $n>0$, and is spanned by the vectors $a_{-n_{1}} \ldots a_{-n_{k}}|0\rangle, n_{1} \geqslant n_{2} \geqslant \ldots \geqslant n_{k}$.
} 
one can get for the generating function of one colored Young diagram (here we imply $\left.k_{0}=k_{p}=0\right)$

$$
\chi_{r}\left(k_{1}, \ldots, k_{p-1} \mid q\right)=q^{\sum_{i=1}^{p-1}\left(k_{i}^{2}+\frac{k_{i}}{p}-k_{i} k_{i+1}\right)-k_{r}} \cdot\left(\chi_{B}(q)\right)^{p} .
$$

It is straightforward to obtain the generating function of the pair of Young diagrams. The generating function of the pair of Young diagrams with the coloring $r_{1}, r_{2}$ and $k_{1}, \ldots, k_{p-1}$ is equal to

$$
\chi_{r_{1}, r_{2}}\left(k_{1}, \ldots, k_{p-1} \mid q\right)=\sum_{\substack{m_{i}+n_{i}=k_{i} \\ i=1, \ldots, p-1}} \chi_{r_{1}}\left(m_{1}, \ldots, m_{p-1} \mid q\right) \chi_{r_{2}}\left(n_{1}, \ldots, n_{p-1} \mid q\right) .
$$

Then using the formula (2.9) one obtains

$$
\begin{aligned}
& \chi_{r_{1}, r_{2}}\left(k_{1}, \ldots, k_{p-1} \mid q\right)= \\
& =\left(\chi_{B}(q)\right)^{2 p} \sum_{m_{1}, \ldots, m_{p-1} \in \mathbb{Z}} q^{\frac{1}{2} \sum_{i=1}^{p-1}\left(\left(2 m_{i}-k_{i}\right)^{2}-\left(2 m_{i}-k_{i}\right)\left(2 m_{i+1}-k_{i+1}\right)+k_{i}^{2}-k_{i} k_{i+1}+\frac{2 k_{i}}{p}\right)-m_{r_{1}}+m_{r_{2}}-k_{r_{2}}} .
\end{aligned}
$$

And therefore we can obviously get

$$
\begin{gathered}
\sum_{k_{1}, \ldots, k_{p-1}=0}^{1} q^{-\frac{1}{2} \sum_{i=1}^{p-1}\left(k_{i}^{2}-k_{i} k_{i+1}+\frac{2 k_{i}}{p}\right)+\frac{1}{2}\left(k_{r_{1}}+k_{r_{2}}\right)} \chi_{r_{1}, r_{2}}\left(k_{1}, \ldots, k_{p-1} \mid q\right)= \\
=\left(\chi_{B}(q)\right)^{2 p} \sum_{\substack{n_{1}, \ldots, n_{p-1} \in \mathbb{Z} \\
n_{0}=n_{p}=0}} q^{\frac{1}{2} \sum_{\sigma=1}^{p-1}\left(n_{\sigma}^{2}-n_{\sigma} n_{\sigma+1}\right)+\frac{1}{2}\left(n_{r_{2}}-n_{r_{1}}\right)}
\end{gathered}
$$

\subsection{Counting of the non equivalent generating functions of the colored Young diagrams}

We call two generating functions equivalent if they differ only by the multiplication of $q$ to some power (by this definition all the generating functions (2.9) are equivalent). Below we show that the whole infinite set of the generating functions (2.11) can be divided into the finite number of classes of equivalence. From (2.11) we conclude that the generating functions have the following symmetries:

- The invariance under the transformation $k_{m} \rightarrow k_{m}+2$ :

$$
\chi_{r_{1}, r_{2}}\left(k_{1}, \ldots, k_{m}+2, \ldots, k_{p-1} \mid q\right)=q^{2 k_{m}-k_{m+1}-k_{m-1}+\frac{2}{p}+\delta_{m, r_{1}}+\delta_{m, r_{2}}} \chi_{r_{1}, r_{2}}\left(k_{1}, \ldots, k_{p-1} \mid q\right),
$$

where $\delta_{m, n}$ is the Kronecker delta.

- The invariance under the permutation $r_{1} \leftrightarrow r_{2}$ :

$$
\chi_{r_{1}, r_{2}}\left(k_{1}, \ldots, k_{p-1} \mid q\right)=\chi_{r_{2}, r_{1}}\left(k_{1}, \ldots, k_{p-1} \mid q\right) .
$$


- The invariance under the change $r_{1}, r_{2} \rightarrow r_{1}+1, r_{2}+1$ :

$$
\begin{aligned}
& \chi_{r_{1}+1, r_{2}+1}\left(k_{1}, \ldots, k_{p-1} \mid q\right)= \\
& \quad=q^{k_{r_{1}}-k_{r_{1}+1}-\frac{r_{2}-r_{1}}{p}} \chi_{r_{1}, r_{2}}\left(k_{1}, \ldots, k_{r_{1}+1}+1, \ldots, k_{r_{2}}+1, \ldots, k_{p-1} \mid q\right),
\end{aligned}
$$

where we assume that $r_{1} \leqslant r_{2}$.

Applying the symmetries (2.13)-(2.15) we conclude that an arbitrary generating function with the coloring $r_{1}, r_{2}$ and $k_{1}, \ldots, k_{p-1}$ is equivalent to one of the generating functions with $r_{1}=0$ and all $k_{m}$ 's equal to 0 or 1 :

$$
\chi_{0, s}\left(k_{1}, \ldots, k_{p-1} \mid q\right)
$$

where $s=0,1, \ldots, p-1$. Therefore we can confine our consideration to the generating functions of the form (2.16).

It can be shown (see appendix A) that for each $s=0, \ldots, p-1$ the generating functions (2.16) are divided into $[s / 2]+[(p-s) / 2]+1$ classes of equivalence. ${ }^{3}$ The first class of equivalence contains the generating functions equivalent to

$$
\chi_{0, s}(0, \ldots, 0 \mid q)
$$

and its cardinality is $\left(\begin{array}{l}p \\ s\end{array}\right)=\frac{p !}{s !(p-s) !}$. For each of the next $[s / 2]$ classes of equivalence it is convenient to choose the representative

$$
\chi_{0, s}(0, \ldots, \stackrel{s-2 l+1}{0,1}, 0,1,0, \ldots, 1,0,1, \stackrel{s}{0}, 0, \ldots, 0 \mid q)
$$

where $l$ takes integer values from 1 to $[s / 2]$, and from $k_{s-2 l+1}$ to $k_{s}$ there are alternating 1 and 0 and all other $k_{i}=0$. The cardinality of the equivalence class with given $l$ is $\left(\begin{array}{c}p \\ s-2 l\end{array}\right)$. And for each of the last $[(p-s) / 2]$ classes of equivalence we choose the representative

$$
\chi_{0, s}(0, \ldots, 0, \stackrel{s}{0}, 1,0,1, \ldots, 0,1, \stackrel{s+2 n-1}{0}, 1,0, \ldots, 0 \mid q)
$$

where $n$ takes the values from 1 to $[(p-s) / 2]$, and from $k_{s}$ to $k_{s+2 n-1}$ there are alternating 0 and 1 and all other $k_{i}=0$. One can easily check that the sum of the cardinalities of the equivalence classes indeed equals to the number of generating functions (2.16) with given $s$

$$
\left(\begin{array}{l}
p \\
s
\end{array}\right)+\sum_{l=1}^{[s / 2]}\left(\begin{array}{c}
p \\
s-2 l
\end{array}\right)+\sum_{n=1}^{[(p-s) / 2]}\left(\begin{array}{c}
p \\
p-s-2 n
\end{array}\right)=2^{p-1}
$$

\footnotetext{
${ }^{3}$ The symbol $[\ldots]$ means integer part of a number, for example $[3 / 2]=1$.
} 
Now we see that arbitrary generating functions of the fixed points on the moduli space is equivalent to one of the generating functions (2.17)-(2.19) and we obtain

$$
\begin{aligned}
\sum_{k_{1}, \ldots, k_{p-1}=0}^{1} q^{-\frac{1}{2} \sum_{i=1}^{p-1}\left(k_{i}^{2}-k_{i} k_{i+1}+\frac{2 k_{i}}{p}\right)+\frac{k_{s}}{2}} \chi_{0, s}\left(k_{1}, \ldots, k_{p-1} \mid q\right)=\left(\begin{array}{c}
p \\
s
\end{array}\right) \chi_{0, s}(0, \ldots, 0 \mid q)+ \\
+\sum_{n=1}^{[s / 2]}\left(\begin{array}{c}
p \\
s-2 n
\end{array}\right) q^{-\frac{n}{2}\left(1+\frac{2}{p}\right)} \chi_{0, s}(0, \ldots, 0,0,1,0, \ldots, 1,0,1, \stackrel{s}{s-2 n+1}, 0, \ldots, 0 \mid q)+ \\
+\sum_{n=1}^{[(p-s) / 2]}\left(\begin{array}{c}
p \\
s+2 n
\end{array}\right) q^{-\frac{n}{2}\left(1+\frac{2}{p}\right)} \chi_{0, s}(0, \ldots, 0,0,0,1,0,1, \ldots, \stackrel{s+2 n-1}{0}, 1,0,0, \ldots, 0 \mid q) .
\end{aligned}
$$

\section{First realization of the algebra $\mathcal{A}(2, p)$}

In the present section we study the realization of the $\mathcal{A}(2, p)$ as the product of $p$ models with Virasoro symmetry. This realization is represented by the arrow 2 on the figure (1.3). Let us start with the definition of $\mathcal{A}(r, p)$

$$
\mathcal{A}(r, p)=\frac{\widehat{\mathfrak{g l}}(n)_{r}}{\widehat{\mathfrak{g l}}(n-p)_{r}} .
$$

One should notice that the definition (3.1) makes sense only for the positive integer values of the parameter $n$, but the usage of the level-rank duality allows us to extend the definition of $\mathcal{A}(r, p)$ to the case of arbitrary complex $n$. Formally multiplying and dividing (3.1) by the algebras $\widehat{\mathfrak{g l}}(n-\sigma+1)_{r}$ with $\sigma=1, \ldots, p$ we can write

$$
\mathcal{A}(r, p) \supset \frac{\widehat{\mathfrak{g l}}(n-p+1)_{r}}{\widehat{\mathfrak{g l}}(n-p)_{r}} \times \ldots \times \frac{\widehat{\mathfrak{g l} l}(n)_{r}}{\widehat{\mathfrak{g l}}(n-1)_{r}} .
$$

Multiplying and dividing each multiplier of (3.2) by $\widehat{\mathfrak{g l}}(1)_{r}$, and using the level-rank duality ${ }^{4}$ we get

$$
\mathcal{A}(r, p) \supset\left(\mathcal{H} \times \frac{\widehat{\mathfrak{s l}}(r)_{1} \times \widehat{\mathfrak{s l}}(r)_{n-p}}{\widehat{\mathfrak{s l}}(r)_{n-p+1}}\right) \times \ldots \times\left(\mathcal{H} \times \frac{\widehat{\mathfrak{s l}}(r)_{1} \times \widehat{\mathfrak{s l}}(r)_{n-1}}{\widehat{\mathfrak{s l}}(r)_{n}}\right)
$$

Note that the cosets $\widehat{\mathfrak{s l}}(r)_{1} \times \widehat{\mathfrak{s l}}(r)_{n-\sigma} / \widehat{\mathfrak{s l}}(r)_{n-\sigma+1}$ for $\sigma=1, \ldots, p$ are the $\mathrm{W}_{r}$ theories with the central charges

$$
c_{\sigma}=1+\frac{\left(r^{2}-1\right)(n-\sigma)}{n-\sigma+r}-\frac{\left(r^{2}-1\right)(n-\sigma+1)}{n-\sigma+r+1} .
$$

In the case of $r=2$ we have for (3.3):

$$
\mathcal{A}(2, p) \supset \mathcal{H}^{p} \times \operatorname{Vir}^{(1)} \times \ldots \times \operatorname{Vir}^{(p)},
$$

\footnotetext{
${ }^{4}$ Here we used the following level-rank duality trick: $\frac{\widehat{\mathfrak{g} l}(k)_{r}}{\widehat{\mathfrak{g l}}(1)_{r} \times \widehat{\mathfrak{g} l}(k-1)_{r}}=\frac{\widehat{\mathfrak{s} l}(r)_{1} \times \widehat{\mathfrak{s}}(r)_{k-1}}{\widehat{\mathfrak{s l}}(r)_{k}}$ and also the isomorphism $\widehat{\mathfrak{g l}}(1)_{r} \cong \mathcal{H}$.
} 
where $\mathcal{H}^{p}=\mathcal{H} \times \ldots \times \mathcal{H}$ and due to GKO correspondence [22] the cosets $\widehat{\mathfrak{s l}}(2)_{1} \times$ $\widehat{\mathfrak{s l}}(2)_{n-\sigma} / \widehat{\mathfrak{s l}}(2)_{n-\sigma+1}$ are the Virasoro algebras $\operatorname{Vir}^{(\sigma)}$ with the following central charges (see appendix B)

$$
c_{\sigma}=1+\frac{3(n-\sigma)}{n-\sigma+2}-\frac{3(n-\sigma+1)}{n-\sigma+3}=1+6\left(Q_{\sigma}\right)^{2},
$$

where $Q_{\sigma}=b_{\sigma}+b_{\sigma}^{-1}$ and $b_{\sigma}^{2}=-\frac{n-\sigma+3}{n-\sigma+2}$ is the parametrization, which will be useful in what follows. It is easy to check that the parameters $b_{\sigma}$ satisfy the following relations

$$
b_{\sigma}^{2}+b_{\sigma+1}^{-2}=-2, \quad \sigma=1, \ldots, p-1 .
$$

Thus, we have $p-1$ equations for $p$ variables. This means that we are able to express these variables in terms of the only one variable $b$

$$
b_{\sigma}^{2}=\frac{(\sigma-1) Q-p b}{\sigma Q-p b}, \quad \sigma=1, \ldots, p .
$$

In the next subsection we enlarge the r.h.s. of (3.5) up to the full $\mathcal{A}(2, p)$ algebra. In order to perform this we add the set of $p-1$ holomorphic currents of spin $1 / 2$ to the $p$ stress-energy tensors.

\subsection{The $p$ models with Virasoro symmetry}

In the case when parameter $n$ in $\mathcal{A}(2, p)=\widehat{\mathfrak{g l}}(n)_{2} / \widehat{\mathfrak{g l} l}(n-p)_{2}$ is a positive integer, the cosets $\widehat{\mathfrak{s l}}(2)_{1} \times \widehat{\mathfrak{s l}}(2)_{n-\sigma} / \widehat{\mathfrak{s l}}(2)_{n-\sigma+1}$ in (3.3) describe the Minimal models $\mathcal{M}(n-\sigma+1 / n-\sigma+2)$ and the arrow 4 of the scheme (1.3) exactly reproduces the correspondence between the Minimal models, which was widely studied in [23]. In this section in the spirit of [23] we construct the first realization of the algebra $\mathcal{A}(2, p)$.

We recall that Virasoro algebra consists of the infinite number of generators $L_{n}, n \in \mathbb{Z}$ satisfying the following commutation relations

$$
\left[L_{n}, L_{m}\right]=(n-m) L_{n+m}+\frac{c}{12}\left(n^{3}-n\right) \delta_{n+m, 0},
$$

where $c$ is the central charge parametrized as $c=1+6 Q^{2}$ with $Q=b+b^{-1}$. We denote the highest weight state of this algebra by $V_{\lambda}$, which is annihilated by $L_{n}$ with $n>0$ and has the conformal dimension

$$
\Delta(\lambda)=\frac{Q^{2}}{4}-\lambda^{2}
$$

The highest weight states of the degenerate representations of the Virasoro algebra are denoted by $V_{m, n} \equiv V_{\lambda_{m, n}}$, where

$$
\lambda_{m, n}=\frac{m b^{-1}+n b}{2}
$$

and their dimension is

$$
\Delta_{m, n}=\frac{Q^{2}}{4}-\lambda_{m, n}^{2}
$$


In addition to $p$ stress-energy tensors $T^{(\sigma)}$ we form the set of $p-1$ holomorphic currents

$$
J^{(\sigma)}(z) \stackrel{\text { def }}{=} V_{1,2}^{(\sigma)}(z) V_{2,1}^{(\sigma+1)}(z), \quad \sigma=1, \ldots, p-1,
$$

where $V_{m, n}^{(\sigma)}$ is the degenerate field primary with respect to $T^{(\sigma)}$. Due to the relation (3.7) for $b_{\sigma}$ 's the left conformal dimension of the current $J^{(\sigma)}(z)$ is

$$
\Delta_{J^{(\sigma)}}=\Delta_{1,2}^{(\sigma)}+\Delta_{2,1}^{(\sigma+1)}=\frac{1}{2}
$$

while the right conformal dimension is zero. It can be checked that the currents $T^{(\sigma)}(z)$ and $J^{(\sigma)}(z)$ generate an associative chiral algebra [23, 24]. We call this algebra the first realization of the $\mathcal{A}(2, p)$.

Now let us turn to the construction of the representations of the $\mathcal{A}(2, p)$. The first requirement for the highest weight state of the algebra is that it has to be primary with respect to $p$ stress tensors $T^{(\sigma)}$. If $V_{\lambda_{\sigma}}^{(\sigma)}$ is the primary state of the $\sigma$-th stress tensor, then evidently the state

$$
V_{\lambda_{1}}^{(1)} V_{\lambda_{2}}^{(2)} \ldots V_{\lambda_{p}}^{(p)}
$$

is primary with respect to all stress tensors.

Consider the OPE of the currents $J^{(\sigma)}(z)$ with the state $V_{\lambda_{1}}^{(1)} \ldots V_{\lambda_{p}}^{(p)}$. From the fusion rules it follows

$$
\begin{aligned}
J^{(\sigma)}(z) V_{\lambda_{1}}^{(1)}(0) \ldots V_{\lambda_{p}}^{(p)}(0) & =\sum_{m_{\sigma}, m_{\sigma+1}= \pm 1} z^{m_{\sigma} \lambda_{\sigma} b_{\sigma}+m_{\sigma+1} \lambda_{\sigma+1} b_{\sigma+1}^{-1} \times} \\
& \times C^{(\sigma)}\left(m_{\sigma}, m_{\sigma+1} ; \lambda_{1}, \ldots, \lambda_{p}\right)\left[V_{\lambda_{1}}^{(1)} \ldots V_{\lambda_{\sigma}+\frac{m_{\sigma} b_{\sigma}}{2}}^{(\sigma)} V_{\lambda_{\sigma+1}+\frac{m_{\sigma+1}}{2 b_{\sigma+1}}}^{(\sigma+1)} \ldots V_{\lambda_{p}}^{(p)}\right],
\end{aligned}
$$

where $C^{(\sigma)}\left(m_{\sigma}, m_{\sigma+1} ; \lambda_{1}, \ldots, \lambda_{p}\right)$ are the structure constants. To reach locality we have to make the projection [23] and keep only two terms in the sum (3.16), say, with $m_{\sigma}=$ $m_{\sigma+1}= \pm 1$ and also impose condition $\lambda_{\sigma} b_{\sigma}+\lambda_{\sigma+1} b_{\sigma+1}^{-1} \in \mathbb{Z}$ or $\mathbb{Z}+1 / 2$. Now modes of $J^{(\sigma)}$, which act on the states of the representation, are correspondingly half-integer or integer

$$
\begin{aligned}
& J^{(\sigma)}(z) V_{\lambda_{1}}^{(1)}(0) \ldots V_{\lambda_{p}}^{(p)}(0)=\sum_{n \in \mathbb{Z}+\frac{1}{2}} z^{n-\frac{1}{2}} J_{n}^{(\sigma)} V_{\lambda_{1}}^{(1)}(0) \ldots V_{\lambda_{p}}^{(p)}(0), \text { if } \quad \lambda_{\sigma} b_{\sigma}+\lambda_{\sigma+1} b_{\sigma+1}^{-1} \in \mathbb{Z}, \\
& J^{(\sigma)}(z) V_{\lambda_{1}}^{(1)}(0) \ldots V_{\lambda_{p}}^{(p)}(0)=\sum_{n \in \mathbb{Z}} z^{n-\frac{1}{2}} J_{n}^{(\sigma)} V_{\lambda_{1}}^{(1)}(0) \ldots V_{\lambda_{p}}^{(p)}(0), \quad \text { if } \quad \lambda_{\sigma} b_{\sigma}+! \lambda_{\sigma+1} b_{\sigma+1}^{-1} \in \mathbb{Z}+\frac{1}{2} .
\end{aligned}
$$

The second requirement is that the state (3.15) is primary for the currents $J^{(\sigma)}$, i.e. it's annihilated by the modes of all currents $J^{(\sigma)}(z)$ with positive numbers

$$
J_{n}^{(\sigma)} V_{\lambda_{1}}^{(1)}(0) \ldots V_{\lambda_{p}}^{(p)}(0)=0, n>0, \sigma=1, \ldots, p-1 .
$$

This condition leads us to the following relation for the Liouville momenta $\lambda_{1}, \lambda_{2}, \ldots, \lambda_{p}$ :

$$
\lambda_{\sigma} b_{\sigma}+\lambda_{\sigma+1} b_{\sigma+1}^{-1}=0
$$


if the modes of the $\sigma$-th current $J^{(\sigma)}(z)$ are half-integer and

$$
\lambda_{\sigma} b_{\sigma}+\lambda_{\sigma+1} b_{\sigma+1}^{-1}= \pm \frac{1}{2}
$$

if these modes are integer. In analogy with the representations of the NSR algebra let us call the primary state to be Neveu-Schwarz with respect to the $\sigma$-th current, if (3.18) holds, and call it to be Ramond with respect to the $\sigma$-th current, if (3.19) with plus or minus sign holds. In what follows we will be interested in the representations, which are NS with respect to all currents $J^{(\sigma)}(z)$, and in the representations, which are $\mathrm{R}$ with respect to one of the currents and NS with respect to all remaining $p-2$ currents $J^{(\sigma)}(z)$.

First we are going to consider the representation, which is NS with respect to all $p-1$ currents $J^{(\sigma)}(z)$. Let us use the following notation for the primary state of this representation

$$
V_{\lambda_{1}^{0}}^{(1)} V_{\lambda_{2}^{0}}^{(2)} \ldots V_{\lambda_{p}^{0}}^{(p)}
$$

where the notation $\lambda_{\sigma}^{0}$ means, that these Liouville momenta are subject to the conditions

$$
\lambda_{\sigma}^{0} b_{\sigma}+\lambda_{\sigma+1}^{0} b_{\sigma+1}^{-1}=0, \quad \sigma=1, \ldots, p-1 .
$$

Thus, we have $p-1$ equations for $p$ variables. It means, that the only one of these variables is independent and the representation can be labelled by the only one variable $\lambda$ and it is convenient to parametrize $\lambda_{\sigma}^{0}$ in the following way

$$
\lambda_{\sigma}^{0}=\frac{\lambda}{\sqrt{(\sigma Q-p b)(p b-(\sigma-1) Q)}}, \quad \sigma=1, \ldots, p,
$$

which is automatically consistent with (3.21). It is easy now to calculate the conformal dimension of the primary state (3.20):

$$
\Delta_{p, 0}(\lambda)=\frac{1}{p}\left(\frac{Q^{2}}{4}-\lambda^{2}\right)
$$

The OPEs of all the currents $J^{(\sigma)}(z)$ with the corresponding primary state are given by

$$
\begin{aligned}
& J^{(\sigma)}(z) V_{\lambda_{1}^{0}}^{(1)}(0) \ldots V_{\lambda_{p}^{0}}^{(p)}(0)= \\
& \quad=\sum_{m= \pm 1} C^{(\sigma)}\left(m, m ; \lambda_{1}, \ldots, \lambda_{p}\right)\left[V_{\lambda_{1}^{0}}^{(1)} \ldots V_{\lambda_{\sigma}^{0}+\frac{m b_{\sigma}}{2}}^{(\sigma)} V_{\lambda_{\sigma+1}^{0}+\frac{m}{2 b_{\sigma+1}}}^{(\sigma+1)} \ldots V_{\lambda_{p}^{0}}^{(p)}\right] .
\end{aligned}
$$

Let us now consider the representations, which are $\mathrm{R}$ with respect to the $s$-th current $J^{(s)}(z)(s=1, \ldots, p-1)$ and NS with respect to all other currents. We denote the $s$-th representation of this type as

$$
V_{\lambda_{1}^{s}}^{(1)} V_{\lambda_{2}^{s}}^{(2)} \ldots V_{\lambda_{p}^{s}}^{(p)}
$$

where

$$
\lambda_{\sigma}^{s} b_{\sigma}+\lambda_{\sigma+1}^{s} b_{\sigma+1}^{-1}=\left\{\begin{array}{cc}
0, & \sigma \neq s \\
-\frac{1}{2}, & \sigma=s .
\end{array}\right.
$$


Of course, we can take $+\frac{1}{2}$ instead of $-\frac{1}{2}$, but this will give us an equivalent representation. Notice, that there exist therefore $p-1$ representations, which are $\mathrm{R}$ with respect to the only one of the currents.

For the $s$-th representation we again have $p-1$ equations (3.26) on $p$ variables $\lambda_{\sigma}^{s}$ and thus the representations of this type can be parametrized by the only one variable. There exists a convenient parametrization of $\lambda_{\sigma}^{s}$ in terms of $\lambda_{\sigma}^{0}(3.22)$, which automatically satisfies (3.26). Introducing the new variables $d_{\sigma}^{s}$

$$
d_{\sigma}^{s}=\left\{\begin{array}{ll}
\frac{1}{p} \sigma(p-s), & \text { if } \quad \sigma \leqslant s \\
\frac{1}{p} s(p-\sigma), & \text { if } \quad \sigma>s
\end{array} \quad, \quad s=0, \ldots, p-1, \quad \sigma=1, \ldots, p\right.
$$

we have for $\lambda_{\sigma}^{s}$ :

$$
\lambda_{\sigma}^{s}=\lambda_{\sigma}^{0}+d_{\sigma-1}^{s} \frac{b_{\sigma}^{-1}}{2}+d_{\sigma}^{s} \frac{b_{\sigma}}{2},
$$

where $\lambda_{\sigma}^{0}$ are given by (3.22). The conformal dimension of the primary state (3.25) is

$$
\Delta_{p, s}(\lambda)=\frac{1}{p}\left(\frac{Q^{2}}{4}-\lambda^{2}\right)+\frac{s(p-s)}{4 p} .
$$

The OPEs of the currents $J^{(\sigma)}(z)$ with the corresponding primary state are given by

$$
\begin{aligned}
& J^{(\sigma)}(z) V_{\lambda_{1}^{s}}^{(1)}(0) \ldots V_{\lambda_{p}^{s}}^{(p)}(0)= \\
& =\sum_{m= \pm 1} C^{(\sigma)}\left(m, m ; \lambda_{1}, \ldots, \lambda_{p}\right)\left[V_{\lambda_{1}^{s}}^{(1)} \ldots V_{\lambda_{\sigma}^{s}+\frac{m b_{\sigma}}{2}}^{(\sigma)} V_{\lambda_{\sigma+1}^{s}+\frac{m}{2 b_{\sigma+1}}}^{(\sigma+1)} \ldots V_{\lambda_{p}^{s}}^{(p)}\right], \sigma \neq s, \\
& J^{(s)}(z) V_{\lambda_{1}^{s}}^{(1)}(0) \ldots V_{\lambda_{p}^{s}}^{(p)}(0)= \\
& =\sum_{m= \pm 1} z^{-\frac{m}{2}} C^{(\sigma)}\left(m, m ; \lambda_{1}, \ldots, \lambda_{p}\right)\left[V_{\lambda_{1}^{s}}^{(1)} \ldots V_{\lambda_{\sigma}^{s}+\frac{m b_{\sigma}}{2}}^{(\sigma)} V_{\lambda_{\sigma+1}^{s}+\frac{m}{2 b_{\sigma+1}}}^{(\sigma+1)} \ldots V_{\lambda_{p}^{s}}^{(p)}\right] .
\end{aligned}
$$

Now we are going to describe the structure of the considered representations and calculate their characters. The states in all representations are generated by the $p$ stress tensors and $p-1$ holomorphic currents. The structure of the OPEs of these currents with the primary states (3.24) and (3.30) tells us that the representation besides the Virasoro descendants of the primary state contains also the states, whose Liouville momenta of the $\sigma$-th and $\sigma+1$-th fields are shifted by $\pm b_{\sigma} / 2$ and $\pm b_{\sigma+1}^{-1} / 2$ respectively and which are also primary with respect to the $p$ stress tensors. Thus, taking the OPE of the current $J^{(\sigma)}(z)$ with these states with shifted momenta $\lambda_{\sigma}$ and $\lambda_{\sigma+1}$ we will generate the infinite number of states, which are primary with respect to the $p$ stress tensors. For the $s$-th representation these states are given by

$$
V_{\lambda_{1}^{s}+n_{1} \frac{b_{1}}{2}}^{(1)} V_{\lambda_{2}^{s}+n_{1} \frac{b_{2}^{-1}}{2}+n_{2} \frac{b_{2}}{2}}^{(2)} \ldots V_{\lambda_{p}^{s}+n_{p-1} \frac{b_{p}^{-1}}{2}}^{(p)},
$$

where $n_{\sigma} \in \mathbb{Z}$ and $n_{0}=n_{p}=0$. In addition, $p$ stress tensors generate the Virasoro submodules from each of (3.31). Therefore, the $s$-th representation of the first realization 
of $\mathcal{A}(2, p)$, which we denote by $\pi_{p, s}^{1}$ is given by the following expression

$$
\pi_{p, s}^{1} \stackrel{\text { def }}{=} \bigoplus_{n_{1}, \ldots, n_{p-1} \in \mathbb{Z}}\left[\begin{array}{l}
\mathrm{V}_{\lambda_{1}^{s}+n_{1} \frac{b_{1}}{2}}^{(1)}
\end{array}\right] \times\left[\begin{array}{l}
\mathrm{V}^{(2)} \\
\lambda_{2}^{s}+n_{1} \frac{b_{2}^{-1}}{2}+n_{2} \frac{b_{2}}{2}
\end{array}\right] \times \ldots \times\left[\begin{array}{l}
\mathrm{V}^{(p)} \\
\lambda_{p}^{s}+n_{p-1} \frac{b_{p}^{-1}}{2}
\end{array}\right],
$$

where $s=0, \ldots, p-1$ and square brackets denote the Virasoro module.

The character of the representation $\pi_{p, s}^{1}$ can be now easily calculated. We have

$$
\chi_{p}^{s}(q)=\operatorname{tr}\left\{q^{\sum_{\sigma=1}^{p} L_{0}^{(\sigma)}}\right\}_{\pi_{p, s}^{1}},
$$

where $L_{0}^{(\sigma)}$ is the element of the Virasoro subalgebra generated by the stress tensor $T^{(\sigma)}$. Thus,

$$
\chi_{p}^{s}(q)=\left(\chi_{B}(q)\right)^{p} \sum_{\substack{n_{1}, \ldots, n_{p-1} \in \mathbb{Z} \\ n_{0}=n_{p}=0}} q^{\sum_{\sigma=1}^{p} \Delta^{(\sigma)}\left(\lambda_{\sigma}^{s}+n_{\sigma-1} \frac{b_{\sigma}^{-1}}{2}+n_{\sigma} \frac{b_{\sigma}}{2}\right)} .
$$

Calculating the sum of conformal dimensions one gets

$$
\chi_{p}^{s}(q)=q^{\Delta_{p, s}(\lambda)}\left(\chi_{B}(q)\right)^{p} \sum_{\substack{n_{1}, \ldots, n_{p-1} \in \mathbb{Z} \\ n_{0}=n_{p}=0}} q^{\frac{1}{2} \sum_{\sigma=1}^{p-1}\left(n_{\sigma}^{2}-n_{\sigma} n_{\sigma+1}\right)+\frac{1}{2} n_{s}},
$$

where $\Delta_{p, s}(\lambda)=\left(Q^{2} / 4-\lambda^{2}\right) / p+s(p-s) /(4 p)$, and $\chi_{B}(q)$ is defined in (2.8).

\subsection{Comparison with the generating functions of the colored Young diagrams}

As it was argued in the Introduction, the reason for the AGT relation is the statement that one can construct the action of the symmetry algebra $\mathcal{A}(2, p)$ on the equivariant cohomologies of the moduli space of instantons $\bigsqcup_{N} \mathcal{M}(2, \mathbb{N})^{\mathbb{Z}_{p}}$. Now we obviously see from (2.12) and (3.35) the coincidence of the generating functions of the fixed points on the moduli space and the characters of the first realization of $\mathcal{A}(2, p)$ :

$$
q^{-\Delta_{p, s}(\lambda)}\left(\chi_{B}(q)\right)^{p} \chi_{p}^{s}(q)=\sum_{k_{1}, \ldots, k_{p-1}=0}^{1} q^{-\frac{1}{2} \sum_{i=1}^{p-1}\left(k_{i}^{2}-k_{i} k_{i+1}+\frac{2 k_{i}}{p}\right)+\frac{k_{s}}{2}} \chi_{0, s}\left(k_{1}, \ldots, k_{p-1} \mid q\right),
$$

where $s=0, \ldots, p-1$ and the generating function $\chi_{0, s}\left(k_{1}, \ldots, k_{p-1}\right)$ is given in $(2.11)$. Note that the obtained identity establishes the correspondence between the characters of the representations of the algebra $\mathcal{A}(2, p)$ in the first realization and the generating functions of the fixed points of the moduli space labelled by the colored Young diagrams. Below we illustrate the obtained identity by listing the examples for $p=2,3,4$. Using the formula (2.21) we find

The $p=2$ case:

$$
\begin{aligned}
& q^{-\Delta_{2,0}(\lambda)}\left(\chi_{B}(q)\right)^{2} \chi_{2}^{0}(q)=\chi_{0,0}(0 \mid q)+q^{-1} \chi_{0,0}(1 \mid q) \\
& q^{-\Delta_{2,1}(\lambda)}\left(\chi_{B}(q)\right)^{2} \chi_{2}^{1}(q)=2 \chi_{0,1}(0 \mid q) .
\end{aligned}
$$


The $p=3$ case:

$$
\begin{aligned}
& q^{-\Delta_{3,0}(\lambda)}\left(\chi_{B}(q)\right)^{3} \chi_{3}^{0}(q)=\chi_{0,0}(0,0 \mid q)+3 q^{-\frac{5}{6}} \chi_{0,0}(1,0 \mid q), \\
& q^{-\Delta_{3,1}(\lambda)}\left(\chi_{B}(q)\right)^{3} \chi_{3}^{1}(q)=3 \chi_{0,1}(0,0 \mid q)+q^{-\frac{5}{6}} \chi_{0,1}(0,1 \mid q) .
\end{aligned}
$$

The $p=4$ case:

$$
\begin{aligned}
& q^{-\Delta_{4,0}(\lambda)}\left(\chi_{B}(q)\right)^{4} \chi_{4}^{0}(q)=\chi_{0,0}(0,0,0 \mid q)+6 q^{-\frac{3}{4}} \chi_{0,0}(1,0,0 \mid q)+q^{-\frac{3}{2}} \chi_{0,0}(1,0,1 \mid q), \\
& q^{-\Delta_{4,1}(\lambda)}\left(\chi_{B}(q)\right)^{4} \chi_{4}^{1}(q)=4 \chi_{0,1}(0,0,0 \mid q)+4 q^{-\frac{3}{4}} \chi_{0,1}(0,0,1 \mid q), \\
& q^{-\Delta_{4,2}(\lambda)}\left(\chi_{B}(q)\right)^{4} \chi_{4}^{2}(q)=6 \chi_{0,2}(0,0,0 \mid q)+2 q^{-\frac{3}{4}} \chi_{0,2}(1,0,0 \mid q) .
\end{aligned}
$$

\section{Second realization of the algebra $\mathcal{A}(2, p)$}

The present section is devoted to the other realization of $\mathcal{A}(2, p)$ as the product of consecutive Minimal models and coset. This relalization is depicted by the arrow 3 on the figure (1.3). Let us again start with the definition of the algebra $\mathcal{A}(r, p)$ :

$$
\mathcal{A}(r, p)=\frac{\widehat{\mathfrak{g l}}(n)_{r}}{\widehat{\mathfrak{g l}}(n-p)_{r}} .
$$

The usage of the level-rank duality allows us to rewrite (4.1) in the following way

$$
\mathcal{A}(r, p) \supset \mathcal{H} \times \widehat{\mathfrak{s l}}(p)_{r} \times \frac{\widehat{\mathfrak{s l}}(r)_{p} \times \widehat{\mathfrak{s l} l}(r)_{n-p}}{\widehat{\mathfrak{s l}}(r)_{n}} .
$$

Utilizing the method of $[23,25]$, i.e. formally multiplying and dividing (4.2) by the algebras $\widehat{\mathfrak{g l}}(k)_{r}$ with $k=2, \ldots, p-1$ we have

$$
\mathcal{A}(r, p) \supset \widehat{\mathfrak{g l}}(1)_{r} \times \frac{\widehat{\mathfrak{g l}}(2)_{r}}{\widehat{\mathfrak{g l}}(1)_{r}} \times \ldots \times \frac{\widehat{\mathfrak{g l}}(p)_{r}}{\widehat{\mathfrak{g l} l}(p-1)_{r}} \times \frac{\widehat{\mathfrak{s l}}(r)_{p} \times \widehat{\mathfrak{s l}}(r)_{n-p}}{\widehat{\mathfrak{s l}}(r)_{n}} .
$$

Then applying the level-rank duality as we did in (3.2) we get

$$
\mathcal{A}(r, p) \supset \mathcal{H}^{p} \times \frac{\widehat{\mathfrak{s l}}(r)_{1} \times \widehat{\mathfrak{s l} l}(r)_{1}}{\widehat{\mathfrak{s l} l}(r)_{2}} \times \ldots \times \frac{\widehat{\mathfrak{s l}}(r)_{1} \times \widehat{\mathfrak{s l} l}(r)_{p-1}}{\widehat{\mathfrak{s l}}(r)_{p}} \times \frac{\widehat{\mathfrak{s l}}(r)_{p} \times \widehat{\mathfrak{s l} l}(r)_{n-p}}{\widehat{\mathfrak{s l} l}(r)_{n}} .
$$

The cosets $\widehat{\mathfrak{s l}}(r)_{1} \times \widehat{\mathfrak{s l}}(r)_{m-1} / \widehat{\mathfrak{s l}}(r)_{m}$ with $m=2, \ldots, p$ are the consecutive Minimal models with the $\mathrm{W}_{r}$-symmetry [26] and with the central charges

$$
c_{r, m}^{\mathrm{MM}}=(r-1)\left(1-\frac{r(r+1)}{(m+r-1)(m+r)}\right), \quad m=2, \ldots, p,
$$

while the coset $\widehat{\mathfrak{s l}}(r)_{p} \times \widehat{\mathfrak{s l}}(r)_{n-p} / \widehat{\mathfrak{s l}}(r)_{p}$ determines the conformal field theory with the central charge

$$
c_{\mathrm{WPF}}=\frac{p\left(r^{2}-1\right)}{p+r}\left(1-\frac{r(p+r)}{(n-p+r)(n+r)}\right) .
$$


In the case of $\mathcal{A}(2, p)$ or $r=2$, the cosets $\widehat{\mathfrak{s l}}(2)_{1} \times \widehat{\mathfrak{s l}}(2)_{m-1} / \widehat{\mathfrak{s l}}(2)_{m}$ are isomorphic to the symmetry algebras of the Minimal models $\mathcal{M}(m+1 / m+2)[22,27]$. And we have ${ }^{5}$

$$
\mathcal{A}(2, p) \supset \frac{\widehat{\mathfrak{s l}}(2)_{p} \times \widehat{\mathfrak{s l}}(2)_{n-p}}{\widehat{\mathfrak{s l}}(2)_{n}} \times \mathcal{M}(3 / 4) \times \ldots \times \mathcal{M}(p+1 / p+2) \times \mathcal{H}^{p} .
$$

Further we will show that the character of a certain sum of the representations of the right hand side of (4.7) coincides with the character of the representation of the first realization of $\mathcal{A}(2, p)$, which means that two realizations of $\mathcal{A}(2, p)$ are consistent. Then, automatically all characters will be equal to the sum of the generating functions of the pairs of colored Young diagrams.

\subsection{Representations of the coset $\widehat{\mathfrak{s l}}(2)_{p} \times \widehat{\mathfrak{s l}}(2)_{n-p} / \widehat{\mathfrak{s l}}(2)_{n}$}

In this section we are going to review the representations of the coset $\widehat{\mathfrak{s l}}(2)_{p} \times$ $\widehat{\mathfrak{s l}}(2)_{n-p} / \widehat{\mathfrak{s l}}(2)_{n}[22,27]$. Let us denote the integrable representation of $\widehat{\mathfrak{s l}}(2)_{p}$ by $\pi_{p, \frac{m}{2}}$, where $0 \leq m \leq p$ and the representation of $\widehat{\mathfrak{s l}}(2)_{n-p}$ by $\pi_{n-p, j}$, where $j$ is a continuous parameter. The representation of the numerator $\pi_{p, \frac{m}{2}} \times \pi_{n-p, j}$ is decomposed into the sum of the irreducible representations of the product of the denominator and coset itself:

$$
\pi_{p, \frac{m}{2}} \otimes \pi_{n-p, j}=\underset{\substack{s \in \mathbb{Z} \\ m-s=0 \bmod 2}}{\bigoplus} \pi_{n, j+\frac{s}{2}} \otimes V_{s}^{m}(p, j)
$$

where $\pi_{n, j+\frac{s}{2}}$ is the representation of the denominator $\widehat{\mathfrak{s l}}(2)_{n}$ and $V_{s}^{m}(p, j)$ is the representation of the coset $\widehat{\mathfrak{s l}}(2)_{p} \times \widehat{\mathfrak{s l}}(2)_{n-p} / \widehat{\mathfrak{s l}}(2)_{n}$.

The characters $c_{s}^{m}(q)$ of the representations of $\widehat{\mathfrak{s l}}(2)_{p} \times \widehat{\mathfrak{s l}}(2)_{n-p} / \widehat{\mathfrak{s l}}(2)_{n}$ are given by the branching functions which can be found from the relation for the characters originating from (4.8):

$$
\chi_{p, \frac{m}{2}}^{\mathfrak{s l}(2)}(q, z) \chi_{n-p, j}^{\mathfrak{s l}(2)}(q, z)=\sum_{\substack{s \in \mathbb{Z} \\ m-s=0 \bmod 2}} \chi_{n, j+\frac{s}{2}}^{\mathfrak{s l}(2)}(q, z) c_{s}^{m}(q) .
$$

These characters are labelled by the integer parameters $m$ and $s$ and continuous parameter $j$ and are given by [30]:

$$
\begin{aligned}
c_{s}^{m}(q)=q^{\delta_{s}^{m}(j)} \chi_{B}^{3}(q) \sum_{r, l=0}^{+\infty}(-1)^{r+l} q^{\frac{l(l+1)}{2}+\frac{r(r+1)}{2}+r l(p+1)} \times \\
\quad \times\left(q^{l \frac{m-s}{2}+r \frac{m+s}{2}}-q^{p+1-m+l\left(p+1-\frac{m-s}{2}\right)+r\left(p+1-\frac{m+s}{2}\right)}\right),
\end{aligned}
$$

where $0 \leqslant m \leqslant p, m-s=0 \bmod 2$, and

$$
\delta_{s}^{m}(j)=\frac{j(j+1)}{n-p+2}+\frac{m(m+2)}{4(p+2)}-\frac{(2 j+s)(2 j+s+2)}{4(n+2)} .
$$

\footnotetext{
${ }^{5}$ The symmetry of the $\mathbb{Z}_{p}$ parafermionic Liouville field theory is described by the coset $\widehat{\mathfrak{s} l}(2)_{p} \times$ $\widehat{\mathfrak{s l}}(2)_{n-p} / \widehat{\mathfrak{s} l}(2)_{n}[12,28,29]$.
} 
Using the formula (4.10) it can be shown that $c_{s}^{m}(q)$ admits the following symmetries

$$
\begin{aligned}
c_{-s}^{m}(q) & =c_{s}^{m}(q), \\
c_{s+2 p}^{m}(q) & =c_{s}^{m}(q) .
\end{aligned}
$$

Thus, we can confine our consideration to $s$, which are integers from 0 to $p$.

Below we are going to pass to the different parametrization of the highest weight state and of the character of the highest weight representation, which is convenient in our calculations. So we pass from the parameters $n$ and $j$ to the $b$ and $\mu$ as follows

$$
b^{2}=-\frac{n+2}{n-p+2}, \quad j=\frac{1}{Q}\left(\mu-\frac{Q}{2}-\frac{s}{2 b}\right), \quad Q=b+b^{-1} .
$$

And we denote the highest weight state of the coset by $\Psi_{s}^{m}(\mu)$ and its representation by $\left[\Psi_{s}^{m}(\mu)\right]$. The dimension of the highest weight state and the character of this representation are given by

$$
\begin{aligned}
& \Delta_{s}^{m}(\mu)= \begin{cases}\frac{1}{p}\left(\frac{Q^{2}}{4}-\mu^{2}\right)+\frac{s(p-s)}{2 p(p+2)}+\frac{(m-s)(m+s+2)}{4(p+2)}, & m \geq s \\
\frac{1}{p}\left(\frac{Q^{2}}{4}-\mu^{2}\right)+\frac{s(p-s)}{2 p(p+2)}+\frac{(s-m)(2 p-m-s+2)}{4(p+2)}, & m<s\end{cases} \\
& c_{s}^{m}(q)=q^{D_{s}^{m}(\mu)} \chi_{B}^{3}(q) \sum_{r, l=0}^{\infty}(-1)^{r+l} q^{\frac{l(l+1)}{2}+\frac{r(r+1)}{2}+r l(p+1)} \times \\
& \times\left(q^{l \frac{m-s}{2}+r \frac{m+s}{2}}-q^{p+1-m+l\left(p+1-\frac{m-s}{2}\right)+r\left(p+1-\frac{m+s}{2}\right)}\right),
\end{aligned}
$$

where $0 \leqslant m, s \leqslant p, m-s=0 \bmod 2$ and

$$
D_{s}^{m}(\mu)=\frac{1}{p}\left(\frac{Q^{2}}{4}-\mu^{2}\right)+\frac{s(p-s)}{2 p(p+2)}+\frac{(m-s)(m+s+2)}{4(p+2)}
$$

for all $m$ and $s$.

\subsection{Product of consecutive Minimal models}

The other main part of (4.7) is the product of the Minimal models. Minimal model $\mathcal{M}(m / m+1)$ has the central charge $c_{2, m}^{\mathrm{MM}}=1-6 /(m(m+1))$ and has the finite set of primary fields $\phi_{r, s}^{(m)}$ with $r=1,2, \ldots m-1$, and $s=1,2, \ldots m$ [31]. The dimensions of these primary fields are given by the formula

$$
h_{r, s}^{(m)}=\frac{((m+1) r-m s)^{2}-1}{4 m(m+1)} .
$$

The following fields are identified with each other $\phi_{r, s}^{(m)}=\phi_{m-r, m+1-s}^{(m)}$. We will denote the irreducible Virasoro representation, built from the highest weight state $\phi_{r, s}^{(m)}$ by $M_{r, s}^{(m)}$. The character of such a representation equals to

$$
\chi_{r, s}^{(m)}(q)=\left.\operatorname{Tr}\left(q^{L_{0}}\right)\right|_{M_{r, s}^{(m)}}=\Delta_{r, s}^{m}(q) \chi_{B}(q)
$$


where

$$
\Delta_{r, s}^{m}(q)=\sum_{k \in \mathbb{Z}}\left(q^{\alpha_{r, s}^{m}(k)}-q^{\alpha_{r,-s}^{m}(k)}\right), \quad \alpha_{r, s}^{m}(k)=\frac{(2 m(m+1) k-s m+r(m+1))^{2}-1}{4 m(m+1)} .
$$

Consider the product of $p-1$ Minimal models highest weight states $\phi_{1 k_{1}}^{(3)} \times \phi_{k_{1} k_{2}}^{(4)} \times$ $\ldots \times \phi_{k_{p-2} n}^{(p+1)}$, where $k_{i}$ runs from 1 to $i+2$ and $n$ runs from 1 to $p+1$. This composite highest weight state has the dimension (hereafter we imply $k_{0}=1, k_{p-1}=n$ )

$$
h_{n}\left(k_{1}, \ldots, k_{p-2}\right)=\sum_{i=1}^{p-1} h_{k_{i-1} k_{i}}^{(i+2)}=\frac{\left(n^{2}-1\right)(p+1)}{4(p+2)}+\frac{1}{2} \sum_{i=0}^{p-2}\left(k_{i}^{2}-k_{i} k_{i+1}\right) .
$$

The irreducible representation which is built from this composite highest weight state is denoted as $M_{1, k_{1}}^{(3)} \times M_{k_{1}, k_{2}}^{(4)} \times \ldots \times M_{k_{p-2}, n}^{(p+1)}$. Below we will consider the following sum of the representations

$$
\bigoplus_{\substack{\left\{k_{1}, \ldots, k_{p-2}\right\} \\ 1 \leq k_{i} \leq i+2}} M_{1, k_{1}}^{(3)} \times M_{k_{1}, k_{2}}^{(4)} \times \ldots \times M_{k_{p-2}, n}^{(p+1)} .
$$

Denote a character of this sum of the representations by

$$
\operatorname{ch}_{n}(q) \stackrel{\text { def }}{=} \sum_{\substack{\left\{k_{1}, \ldots, k_{p-2}\right\} \\ 1 \leq k_{i} \leq i+2, k_{p-1}=n}} \prod_{i=1}^{p-1} \chi_{k_{i-1} k_{i}}^{(i+2)}(q) .
$$

In the next subsection we will show that the characters of the product of consecutive Minimal models and the coset coincide with the characters of the first realization of $\mathcal{A}(2, p)$.

\subsection{Comparison with the first realization of $\mathcal{A}(2, p)$}

Let us start by considering the following sum of the representations of the coset and consecutive Minimal models

$$
\left[\Psi_{s}^{m}(\mu)\right] \times \bigoplus_{\substack{\left\{k_{1}, \ldots, k_{p-2}\right\} \\ 1 \leqslant k_{i} \leqslant i+2}} M_{1, k_{1}}^{(3)} \times M_{k_{1}, k_{2}}^{(4)} \times \ldots \times M_{k_{p-2}, n}^{(p+1)}
$$

where $1 \leqslant n \leqslant p+1,0 \leqslant m, s \leqslant p$ with $m-s=0 \bmod 2$. The character of such a representation is equal to

$$
c_{s}^{m}(q) \operatorname{ch}_{n}(q) .
$$

Note, that the representation (4.22) is labelled by three integer parameters $0 \leq m, s \leq p$, $m-s=0 \bmod 2,1 \leq n \leq p+1$ and one continuous parameter $\mu$. Remember that we are looking for the sum of the representations of the form (4.22), character of which coincides with the character $\chi_{p}^{s}(q)$ of the representation of the first realization of $\mathcal{A}(2, p)$, which is labelled by the integer parameter $0 \leq s \leq p-1$ and continuous parameter $\lambda$.

The character (3.35) of the representation of $p$ models $\chi_{p}^{s}(q)$ looks like $q^{\Delta_{p, s}(\lambda)}$ multiplied by some series containing integer and half-integer powers of $q$ and the character (4.23) 
is the sum over $k_{i}$ 's of $q^{\Delta_{s}^{m}(\lambda)+h_{n}\left(k_{1}, \ldots, k_{p-2}\right)}$ multiplied by the series containing integer powers. Because $h_{n}\left(k_{1}, \ldots, k_{p-2}\right)-h_{n}\left(k_{1}^{\prime}, \ldots, k_{p-2}^{\prime}\right) \in \mathbb{Z} / 2$, the necessary condition for the characters to coincide will be

$$
\Delta_{s}^{m}(\mu)+h_{n}\left(k_{1}, \ldots, k_{p-2}\right)-\Delta_{p, s}(\lambda) \in \mathbb{Z} / 2
$$

Performing some algebra we get

$$
\frac{\lambda^{2}-\mu^{2}}{4 p}+\frac{(m+1)^{2}-n^{2}}{4(p+2)}-\frac{s}{4}+\frac{1}{2} \sum_{i=0}^{p-2}\left(k_{i}^{2}-k_{i} k_{i+1}\right) \in \mathbb{Z} / 2 .
$$

Because the left hand side of the expression must be half integer and must not depend on any continuous parameter it is natural to assume $\mu=\lambda$. This leads to the following Diophantine equation

$$
\frac{(m+1)^{2}-n^{2}}{4(p+2)}-\frac{s}{4}+\frac{1}{2} \sum_{i=0}^{p-2}\left(k_{i}^{2}-k_{i} k_{i+1}\right) \in \mathbb{Z} / 2
$$

from which it follows that

$$
\frac{(m-n+1)(m+n+1)}{p+2} \in \mathbb{Z}
$$

Because $-p \leqslant m-n+1 \leqslant p$ and $2 \leqslant m+n+1 \leqslant p+2$, there exist two possibilities

$$
n=m+1, \quad n=p-m+1 .
$$

Therefore, taking into account (4.28), we conclude, that we should take only the representations of the coset and product of Minimal models, which have the form ${ }^{6}$

$$
\begin{aligned}
& {\left[\Psi_{s}^{m}(\lambda)\right] \times \bigoplus_{\substack{\left\{k_{1}, \ldots, k_{p-2}\right\} \\
1 \leqslant k_{i} \leqslant i+2}} M_{1, k_{1}}^{(3)} \times M_{k_{1}, k_{2}}^{(4)} \times \ldots \times M_{k_{p-2}, m+1}^{(p+1)},} \\
& {\left[\Psi_{s}^{m}(\lambda)\right] \times \bigoplus_{\substack{\left\{k_{1}, \ldots, k_{p-2}\right\} \\
1 \leqslant k_{i} \leqslant i+2}} M_{1, k_{1}}^{(3)} \times M_{k_{1}, k_{2}}^{(4)} \times \ldots \times M_{k_{p-2}, p-m+1}^{(p+1)} .}
\end{aligned}
$$

Therefore the representation of the algebra $\mathcal{A}(2, p)$ in the second realization is

$$
\pi_{p, s}^{2}=\bigoplus_{\substack{0 \leqslant m \leqslant p \\ m-s=0 \text { mod } 2}}\left[\Psi_{s}^{m}(\lambda)\right] \times \bigoplus_{\substack{\left\{k_{1}, \ldots, k_{p-2}\right\} \\ 1 \leqslant k_{i} \leqslant i+2}} M_{1, k_{1}}^{(3)} \times M_{k_{1}, k_{2}}^{(4)} \times \ldots \times\left(M_{k_{p-2}, m+1}^{(p+1)} \oplus M_{k_{p-2}, p-m+1}^{(p+1)}\right)
$$

\footnotetext{
${ }^{6}$ These two solutions (4.28) for $n$ lead to the following equations for $k_{i}$ 's

$$
\sum_{i=0}^{p-2}\left(k_{i+1}-k_{i}\right)^{2}=\left\{\begin{array}{lll}
s, & \text { if } & n=m+1 \\
p+s-2 m & \text { if } & n=p-m+1
\end{array}\right.
$$
}


The character of this representation is

$$
\sum_{\substack{0 \leqslant m \leqslant p \\ m-s=0 \text { mod } 2}} c_{s}^{m}(q)\left(\operatorname{ch}_{m+1}(q)+\operatorname{ch}_{p-m+1}(q)\right) .
$$

The conjecture that the representation $\pi_{p, s}^{2}$ is the another form of representation of $\mathcal{A}(2, p)$, i.e.

$$
\pi_{p, s}^{1} \cong \pi_{p, s}^{2}
$$

leads us to the following non-trivial identity:

$$
\begin{aligned}
& \sum_{\substack{0 \leqslant m \leqslant p \\
m-s=0 \text { mod } 2}} c_{s}^{m}(q)\left(\operatorname{ch}_{m+1}(q)+\operatorname{ch}_{p-m+1}(q)\right)= \\
& =q^{\Delta_{p, s}(\lambda)}\left(\chi_{B}(q)\right)^{p} \sum_{\substack{n_{1}, \ldots, n_{p-1} \in \mathbb{Z} \\
n_{0}=n_{p}=0}} q^{\frac{1}{2} \sum_{\sigma=1}^{p-1}\left(n_{\sigma}^{2}-n_{\sigma} n_{\sigma+1}\right)+\frac{1}{2} n_{s}},
\end{aligned}
$$

where expressions for the characters are given in (3.35), (4.15), (4.21). We have checked the equality (4.33) for the cases $p=2, \ldots, 8$ order by order up to $q^{6}$. The equality (4.33) proves the consistence of the representations of the algebra $\mathcal{A}(2, p)$ in the first and secondrealizations. It should be noted that in [32] it was shown, that for the case when the equivariant parameter $n=1$ (and in principal for all $n \in \mathbb{Z}$ ), the characters of the representations of the second realization of $\mathcal{A}(2, p)$ can be rewritten as certain series using Generalized Rogers-Ramanujan identities.

\section{Comparison of the instanton partition functions}

There exist two approaches to the calculation of the instanton partition functions in the $\mathcal{N}=2$ supersymmetric gauge theory on $\mathbb{C}^{2} / \mathbb{Z}_{p}$. The difference in these approaches is in the compactification of the instanton moduli space. As the result one gets different expressions for the same instanton partition function. The first compactification of the moduli space is related to the first realization of the algebra $\mathcal{A}(2, p)$. However, unfortunately, we are not aware of any geometrical interpretation for the second realization of $\mathcal{A}(2, p)$.

\subsection{First compactification}

One approach to the calculation of the instanton partition function on $\mathbb{C}^{2} / \mathbb{Z}_{p}$ is the integration over the moduli space of instantons $\bigsqcup_{N} \mathcal{M}\left(X_{p}, r, N\right)$ on the resolved space $X_{p}=\widetilde{\mathbb{C}^{2} / \mathbb{Z}_{p}}$. The partition function in this approach was calculated in [33, 34]:

$$
\begin{aligned}
& Z_{\text {inst }}^{p, s}\left(\vec{a}, \epsilon_{1}, \epsilon_{2} \mid \Lambda\right)= \\
& \quad=\sum_{\substack{n_{1}, \ldots, n_{p-1} \in \mathbb{Z} \\
n_{0}=n_{p}=0}} \frac{\Lambda^{\left(n_{i}+d_{i}^{s}\right) C_{i j}\left(n_{j}+d_{j}^{s}\right)}}{l_{p, s}^{\mathrm{vec}}\left(a, n_{1}, \ldots, n_{p-1}\right)} \prod_{\sigma=1}^{p} Z_{\text {inst }}^{\mathbb{C}^{2}}\left(\vec{a}_{s}^{(\sigma)}, \epsilon_{1}^{(\sigma)}, \epsilon_{2}^{(\sigma)} \mid \Lambda\right), \quad s=0, \ldots, p-1,
\end{aligned}
$$


where $\vec{a}=(a,-a), \vec{a}_{s}^{(\sigma)}=\left(a_{s}^{(\sigma)},-a_{s}^{(\sigma)}\right)$, and $a_{s}^{(\sigma)}=a+\left(n_{\sigma+1}+d_{\sigma+1}^{s}\right) \epsilon_{1}^{(\sigma)}+\left(n_{\sigma}+d_{\sigma}^{s}\right) \epsilon_{2}^{(\sigma)}$, and regularization parameters are $\epsilon_{1}^{(\sigma)}=(p-\sigma) \epsilon_{1}-\sigma \epsilon_{2}, \epsilon_{2}^{(\sigma)}=(\sigma+1-p) \epsilon_{1}+(\sigma+1) \epsilon_{2}$. The shifts $d_{\sigma}^{s}$ are given by the formula (3.27):

$$
d_{\sigma}^{s}=\left\{\begin{array}{ll}
\frac{1}{p} \sigma(p-s), & \text { if } \quad \sigma \leqslant s \\
\frac{1}{p} s(p-\sigma), & \text { if } \quad \sigma>s
\end{array} \quad, \quad s=0, \ldots, p-1, \quad \sigma=1, \ldots, p .\right.
$$

And $C_{i j}$ is the $(p-1) \times(p-1)$ Cartan matrix of the simple Lie algebra $A_{p-1}$.

The $\mathrm{SU}(2)$ instanton partition function on $\mathbb{C}^{2}$ was calculated in [35]:

$$
Z_{\text {inst }}^{\mathbb{C}^{2}}\left(\vec{a}, \epsilon_{1}, \epsilon_{2} \mid \Lambda\right)=\sum_{\left(Y_{1}, Y_{2}\right)} \Lambda^{\left|Y_{1}\right|+\left|Y_{2}\right|} \prod_{i, j=1}^{2} \prod_{s \in Y_{i}} \frac{1}{E_{Y_{i}, Y_{j}}\left(s \mid a_{i}-a_{j}\right)\left(\epsilon_{1}+\epsilon_{2}-E_{Y_{i}, Y_{j}}\left(s \mid a_{i}-a_{j}\right)\right)},
$$

where $|Y|$ is the total number of the boxes in the Young diagram $Y, s$ denotes a box in the Young diagram $Y$, and

$$
E_{Y, W}(a \mid s)=a-l_{W}(s) \epsilon_{1}+\left(a_{Y}(s)+1\right) \epsilon_{2},
$$

where $a_{Y}(s)$ and $l_{Y}(s)$ is the arm and leg length respectively, i.e. the number of boxes in $Y$ to the right and below of the box $s \in Y$.

The functions $l_{p, s}^{\mathrm{vec}}\left(a, n_{1}, \ldots, n_{p-1}\right)$ are called blow-up factors and were calculated by geometrical methods in [34] and are given by

$$
\begin{aligned}
l_{p, s}^{\mathrm{vec}}\left(a, n_{1}, \ldots, n_{p-1}\right)= & \prod_{\sigma=0}^{p-1} g^{(\sigma)}\left(2 a_{s}^{(\sigma)}, \epsilon_{1}^{(\sigma)}, \epsilon_{2}^{(\sigma)}, n_{\sigma}+d_{\sigma}^{s}, n_{\sigma+1}+d_{\sigma+1}^{s}\right) \times \\
& \times g^{(\sigma)}\left(-2 a_{s}^{(\sigma)}, \epsilon_{1}^{(\sigma)}, \epsilon_{2}^{(\sigma)},-\left(n_{\sigma}+d_{\sigma}^{s}\right),-\left(n_{\sigma+1}+d_{\sigma+1}^{s}\right)\right),
\end{aligned}
$$

where

$$
g^{(\sigma)}\left(a, e_{1}, e_{2}, \mu, \nu\right)=\left\{\begin{array}{clc}
\prod_{\substack{m \geqslant 0, n \leqslant-1 \\
\sigma(\nu+m) \leqslant(\sigma+1)(\mu+n)}}\left(a+m e_{1}+n e_{2}\right), & \text { if } & \sigma \nu<(\sigma+1) \mu \\
1, & \text { if } & \sigma \nu=(\sigma+1) \mu . \\
\prod_{\substack{m \leqslant-1, n \geqslant 0 \\
\sigma(\nu+m)>(\sigma+1)(\mu+n)}}\left(a+m e_{1}+n e_{2}\right), & \text { if } & \sigma \nu>(\sigma+1) \mu
\end{array}\right.
$$

\subsection{Second compactification}

The other compactification of the instanton moduli space is obtained by the lift of the action of $\mathbb{Z}_{p}$ group in $\mathbb{C}^{2} / \mathbb{Z}_{p}$ to the moduli space $\bigsqcup_{N} \mathcal{M}(2, \mathbb{N})$ on $\mathbb{C}^{2}$. The resulting moduli space is denoted by $\bigsqcup_{N} \mathcal{M}(2, \mathbb{N})^{\mathbb{Z}_{p}}$ and its fixed points are labelled by the pairs of Young diagrams with $p$ colors. Thus, in the instanton partition function corresponding to this compactification of the moduli space we take the sum only over these Young diagrams, and also count only the special boxes of these Young diagrams. So as in the section 2 we take the sum over set $\diamond$ of the pairs of Young diagrams $\left(Y_{1}, Y_{2}\right)$ :

$$
\diamond=\left\{\left(Y_{1}, Y_{2}\right) \mid \begin{array}{l|l|l|l|l|l|l|l|l|}
r_{1} & & r_{2}
\end{array}, \sharp\left([m)-\sharp\left([0)=k_{m}\right\},\right.\right.
$$


where the box in $Y_{1}$ with the coordinates $(i, j)$ has the color $r_{1}+i-j \bmod p$ and the box $(i, j)$ in $Y_{2}$ has the color $r_{2}+i-j \bmod p$ and $\sharp(m), \sharp(0)$ - the numbers of the boxes in $\left(Y_{1}, Y_{2}\right)$ with $m$ and 0 color respectively.

Also introduce the formula $[14,36,37]$

$$
\begin{aligned}
\mathcal{Z}_{r_{1}, r_{2}}\left(k_{1}, \ldots, k_{p-1}\left|\vec{a}, \epsilon_{1}, \epsilon_{2}\right| \Lambda\right) & = \\
& =\sum_{\left(Y_{1}, Y_{2}\right) \in \diamond} \Lambda^{\frac{\left|Y_{1}\right|+\left|Y_{2}\right|}{p}} \prod_{i, j=1}^{2} \prod_{s \in Y_{i}}^{\tilde{E}} \frac{1}{E_{Y_{i}, Y_{j}}\left(s \mid a_{i}-a_{j}\right)\left(\epsilon_{1}+\epsilon_{2}-E_{Y_{i}, Y_{j}}\left(s \mid a_{i}-a_{j}\right)\right)},
\end{aligned}
$$

where the product $\tilde{\prod}$ goes only through $s \in Y_{i}$ that satisfy $l_{Y_{j}}(s)+a_{Y_{i}}(s)+1 \equiv r_{j}-r_{i} \bmod p$. After all the notations being introduced we can present the expression for the instanton partition function in the second compactification:

$$
Z_{\text {inst }}^{p, s}\left(\vec{a}, \epsilon_{1}, \epsilon_{2} \mid \Lambda\right)=\sum_{k_{1}, \ldots, k_{p-1}=0}^{1} \Lambda^{-\frac{1}{2} \sum_{i=1}^{p-1}\left(k_{i}^{2}-k_{i} k_{i+1}+\frac{2 k_{i}}{p}\right)+\frac{k_{s}}{2}} \mathcal{Z}_{0, s}\left(k_{1}, \ldots, k_{p-1}\left|\vec{a}, \epsilon_{1}, \epsilon_{2}\right| \Lambda\right) .
$$

The two expressions for the instanton partition functions (5.1) and (5.9) coincide, as it was checked in $[34,38]$. In the next subsection we give arguments in favor of this equality from the conformal field theory point of view.

\subsection{Bases in conformal field theories and the equality of instanton partition functions}

For the cases $r=2, p=1[39,40]$ and $r=2, p=2[8]$ it was shown that the instanton partition function of the $\mathcal{N}=2$ supersymmetric gauge theory without matter is equal to the norm of the Whittaker vector. In the mentioned cases $r=2$ and $p=1,2$ this Whittaker vector is determined as the eigenvector of the upper nilpotent subalgebra of the symmetry algebra (Virasoro in $p=1$ case and Neveu-Schwarz-Ramond in $p=2$ case). In a situation with arbitrary $p$ and $r=2$ the analogue of the Virasoro and NSR algebras is the coset

$$
\frac{\widehat{\mathfrak{s l}}(2)_{p} \times \widehat{\mathfrak{s l}}(2)_{n-p}}{\widehat{\mathfrak{s l}}(2)_{n}} .
$$

Thus, let us assume that for arbitrary $p$ the Whittaker vector is the eigenvector of the upper nilpotent part of the coset (5.10). Note, that we suppose the remaining part of the $\mathcal{A}(2, p)$ algebra to act by zero on this Whittaker vector. Thus, we assume that for general $p$ we can represent partition function as the norm of the Whittaker vector $|W\rangle$

$$
Z_{\text {inst }}=\langle W \mid W\rangle \text {. }
$$

As it was mentioned in Introduction, there exist two ways to construct the moduli space of instantons for the $\mathcal{N}=2$ supersymmetric $\mathrm{U}(r)$ gauge theory on $\mathbb{C}^{2} / \mathbb{Z}_{p}$. And for each way of compactification of the moduli space there is a basis of geometrical origin, which is in one-to-one correspondence with the fixed points of the torus action. Thus, to calculate the instanton partition function we can use the basis labelled by the colored 
Young diagram, which was constructed explicitly in [20] for the cases $r=1,2$ and $p=2$, or labelled by the $p r$-tuples of ordinary Young diagrams, which was constructed explicitly in $[8,19]$ for $r=2$ and $p=1,2$. Despite for $r=2$ and arbitrary $p$ the basis for both compactifications of the moduli space was not constructed, we assume its existence.

Inserting the complete set of states in the norm of the Whittaker vector in each basis we can establish the equality between the instanton partition functions for the pure gauge theory calculated for the different compactifications of the moduli space. Note that we have already established the correspondence between the fixed points of the moduli space in different compactifications, or, equivalently, between the two bases of geometrical origin corresponding to these compactifications. The form of this correspondence is given by the identity (3.36). Thus, taking the basis vectors corresponding to the fixed points labelled by the Young diagrams with $p$ colors from one side and taking the basis vectors corresponding to the fixed points in another compactification labelled by the $p r$-tuples of Young diagrams and $p-1 r$-dimensional vectors from the other side, after inserting the full set of states in the norm of the Whittaker vector we obtain the formula connecting the instanton partition functions in different compactifications.

\section{Acknowledgments}

The authors are grateful to M. Bershtein, B. Feigin, D. Gepner, L. Spodyneiko, A. Zamolodchikov for useful discussions and interest to our work. M.A. thanks the Simons Center for Geometry and Physics for the kind hospitality during the early stage of this project.

This work was supported by RFBR grants No.12-01-00836-a, 12-02-01092-a, 12-0131236-mol a, 12-02-33011-mol a ved and by the Russian Ministry of Education and Science under the grants 2012-1.5-12-000-1011-012, contract No.8528, 2012-1.1-12-000-1011-016, contract No.8410 and agreement No.14.A18.21.2027. The work of M.A. and G.T. were also supported by the 2012 Dynasty Foundation Grant and Federal Targeted Programs of the Russian Ministry of Education and Science. The research leading to these results has received funding from the People Programme (Marie Curie Actions) of the European Union's Seventh Framework Programme FP7/2007-2013/ under REA Grant Agreement No 317089.

\section{A Symmetries of the generating functions}

The aim of the present appendix is to analyze the symmetries of the generating function of Young diagrams $\chi_{0, s}\left(k_{1}, \ldots, k_{p-1} \mid q\right)$ with $s=0,1, \ldots, p-1$ and $k_{i}$ equal to 0 or 1 . Let us remember the formula (2.11) for the generating function and write it in a more convenient form for the further considerations

$$
\begin{aligned}
\chi_{0, s}\left(k_{1}, \ldots, k_{p-1} \mid q\right)=\left(\chi_{B}(q)\right)^{2 p} q^{\frac{1}{2} \sum_{i=1}^{p-1}\left(k_{i}^{2}-k_{i} k_{i+1}+\frac{2 k_{i}}{p}\right)-\frac{1}{2} k_{s}} \times \\
\times \sum_{\left\{m_{i}\right\} \in \mathbb{Z}} q^{\frac{1}{4} \sum_{i=0}^{s-1}\left(2 m_{i+1}-2 m_{i}-k_{i+1}+k_{i}+1\right)^{2}+\frac{1}{4} \sum_{i=s-1}^{p-1}\left(2 m_{i+1}-2 m_{i}-k_{i+1}+k_{i}\right)^{2}-\frac{s}{4}} .
\end{aligned}
$$


Taking some $j \neq s$ and assuming $k_{j}=0$ and $k_{j-1}=0, k_{j+1}=1$, we make the substitution of the summation variable $m_{j}$ in (A.1)

$$
m_{j}=m_{j+1}+m_{j-1}-\tilde{m}_{j}
$$

which effectively leads to $k_{j}=0 \rightarrow k_{j}=1$. After some calculations, we have

$$
\chi_{0, s}(\ldots, 0, \stackrel{j}{0}, 1, \ldots \mid q)=q^{-\frac{1}{p}} \chi_{0, s}(\ldots, 0, \stackrel{j}{1}, 1, \ldots \mid q), j \neq s .
$$

The same substitution as in (A.2) proves that

$$
\chi_{0, s}(\ldots, 1, \stackrel{j}{0}, 0, \ldots \mid q)=q^{-\frac{1}{p}} \chi_{0, s}(\ldots, 1, \stackrel{j}{1}, 0, \ldots \mid q), j \neq s .
$$

Next we have to consider the situation when $k_{s}=0$. Assuming that $k_{s-1}=0$ and $k_{s+1}=0$, we make the substitution for the summation variable $m_{s}$ in (A.1)

$$
m_{s}=m_{s+1}+m_{s-1}-\tilde{m}_{s}
$$

which effectively leads to $k_{s}=0 \rightarrow k_{s}=1$. After some calculations, we have

$$
\chi_{0, s}(\ldots, 0, \stackrel{s}{0}, 0, \ldots \mid q)=q^{-\frac{1}{p}} \chi_{0, s}(\ldots, 0, \stackrel{s}{1}, 0, \ldots \mid q) .
$$

If $k_{s-1}=1$ and $k_{s+1}=1$ the suitable substitution would be

$$
m_{s}=m_{s+1}+m_{s-1}-\tilde{m}_{s}-1
$$

which again effectively leads to $k_{s}=0 \rightarrow k_{s}=1$. After some calculations, we have

$$
\chi_{0, s}(\ldots, 1, \stackrel{s}{0}, 1, \ldots \mid q)=q^{1-\frac{1}{p}} \chi_{0, s}(\ldots, 1, \stackrel{s}{1}, 1, \ldots \mid q) .
$$

The next task is to determine the classes of inequivalent generating functions for each $s$ with $k_{i}$ equal to 0 or 1 .

Let us start from the case $s=0$. As we remember, the array of $k_{i}$ is a series of $p-1$ zeros and unities. We can look at this array as on the islands of unities in the sea of zeros. Then, one can easily see that the symmetries (A.3) and (A.4) forbid the islands to merge (at least one 0 must be between them), but allows them to change their size. Thus, the class of equivalence is determined by the number of islands $n$, which takes the values $0,1,2, \ldots,\left[\frac{p}{2}\right]$. It is convenient to choose the following representative of the $n$-th class

$$
\chi_{0,0}(1,0,1,0, \ldots, 1,0,1,0, \ldots, 0 \mid q) .
$$

Thus, for $s=0$ the cardinality of the $n$-th class is equal to $\left(\begin{array}{c}p \\ 2 n\end{array}\right)$ (the number of ways to distribute $2 n$ borders of the islands between $p$ places).

Now we proceed with the same calculation for the case $s>0$. Looking again onto the array of $k_{i}$, which consists of 0 and 1 , we notice, that if there is an island of identities, containing the position number $s$, we can destroy this island obtaining the generating function equivalent to $\chi_{0, s}$. Then, due to the symmetries (A.6) and (A.8) we can annihilate 
the islands to left of the position $s$ with the islands to the right of the position $s$. This means, that at the end we will be left with some number of the islands on the one side (left or right) only. This lead us to the conclusion that the class of equivalence in this case is determined by the difference of the number of islands to the left and to the right of the position $s$. Therefore, the number of the classes of equivalence is equal to

$$
\left[\frac{s}{2}\right]+\left[\frac{p-s}{2}\right]+1
$$

Let $l$ be the difference of the number of islands to the left and to the right of $s$-th position. Then, the number of the generating functions in the corresponding class of equivalence with $k_{s}=0$ is given by

$$
\sum_{j=0}^{\left[\frac{s}{2}\right]-l}\left(\begin{array}{c}
s \\
2 n+2 j
\end{array}\right)\left(\begin{array}{c}
p-s \\
2 j
\end{array}\right)
$$

The number of the generating functions in the same class with $k_{s}=1$ (which effectively leads to the addition of one island border on the each side) is given by

$$
\sum_{j=0}^{\left[\frac{s}{2}\right]-l}\left(\begin{array}{c}
s \\
2 l+2 j+1
\end{array}\right)\left(\begin{array}{c}
p-s \\
2 j+1
\end{array}\right) .
$$

Summing up both contributions, we obtain using the Vandermonde's identity

$$
\sum_{j=0}^{\left[\frac{s}{2}\right]-n}\left(\left(\begin{array}{c}
s \\
2 l+2 j
\end{array}\right)\left(\begin{array}{c}
p-s \\
2 j
\end{array}\right)+\left(\begin{array}{c}
s \\
2 l+2 j+1
\end{array}\right)\left(\begin{array}{c}
p-s \\
2 j+1
\end{array}\right)\right)=\left(\begin{array}{c}
p \\
s-2 l
\end{array}\right) .
$$

A convenient choice of the representative of the $l$-th class is

$$
\chi_{0, s}(0, \ldots, \stackrel{s-2 l+1}{0,1}, 0,1,0, \ldots, 1,0,1, \stackrel{s}{0}, 0, \ldots, 0) .
$$

The situation is the same when we have $n$ islands on the right side, except for we should replace $s$ by $p-s$, which gives the cardinality $\left(\begin{array}{c}p \\ p-s-2 n\end{array}\right)$. A convenient representative would be

$$
\chi_{0, s}(0, \ldots, 0, \stackrel{s}{0}, 1,0,1, \ldots 0,1 \stackrel{s+2 n-1}{0}, 1,0, \ldots, 0) .
$$

\section{B Conformal field theories based on the coset}

In the present appendix we give some information about the conformal field theories based on the coset

$$
\frac{\widehat{\mathfrak{s l}}(r)_{l_{1}} \times \widehat{\mathfrak{s l}}(r)_{l_{2}}}{\widehat{\mathfrak{s l}}(r)_{l_{1}+l_{2}}}
$$

In the case of general integer $r \geq 2$ and arbitrary complex $l_{1}$ and $l_{2}$ the coset (B.1) describes the conformal field theory with the central charge

$$
c\left(r, l_{1}, l_{2}\right)=\left(r^{2}-1\right)\left(\frac{l_{1}}{r+l_{1}}+\frac{l_{2}}{r+l_{2}}-\frac{l_{1}+l_{2}}{r+l_{1}+l_{2}}\right) .
$$


In the case $l_{1}=1$ we have the conformal field theory with the central charge

$$
c\left(r, 1, l_{2}\right)=(r-1) \frac{l_{2}\left(2 r+l_{2}+1\right)}{\left(r+l_{2}\right)\left(r+l_{2}+1\right)},
$$

which has the $W_{r}$-symmetry [41]. Then, if $l_{2}$ is a positive integer, the central charge is given by the same formula (B.3) and coset describes the Minimal Model with the $W_{r}$-symmetry.

Let us now consider the case of rank $r=2$, which is studied in the present paper. The coset (B.1) takes the form

$$
\frac{\widehat{\mathfrak{s l}}(2)_{l_{1}} \times \widehat{\mathfrak{s l}}(2)_{l_{2}}}{\widehat{\mathfrak{s l}}(2)_{l_{1}+l_{2}}} .
$$

In the case of arbitrary complex $l_{1}$ and $l_{2}$ the coset (B.4) describes the conformal field theory with the central charge

$$
c\left(2, l_{1}, l_{2}\right)=3\left(\frac{l_{1}}{l_{1}+2}+\frac{l_{2}}{l_{2}+2}-\frac{l_{1}+l_{2}}{l_{1}+l_{2}+2}\right) .
$$

In the case $l_{1}=1$ we have the conformal field theory with the central charge

$$
c\left(2,1, l_{2}\right)=\frac{l_{2}\left(l_{2}+5\right)}{\left(l_{2}+2\right)\left(l_{2}+3\right)}=1-\frac{6}{\left(l_{2}+2\right)\left(l_{2}+3\right)},
$$

which has the Virasoro symmetry. Then, if $l_{2}$ is a positive integer, the central charge is given by the same formula (B.6) and the coset describes the Minimal Model $\mathcal{M}\left(l_{2}+1 / l_{2}+2\right)$, as it was shown in $[22,27]$.

Open Access. This article is distributed under the terms of the Creative Commons Attribution License which permits any use, distribution and reproduction in any medium, provided the original author(s) and source are credited.

\section{References}

[1] L.F. Alday, D. Gaiotto and Y. Tachikawa, Liouville Correlation Functions from Four-dimensional Gauge Theories, Lett. Math. Phys. 91 (2010) 167 [arXiv:0906.3219] [INSPIRE].

[2] N. Wyllard, $A(N-1)$ conformal Toda field theory correlation functions from conformal $N=2$ $\mathrm{SU}(N)$ quiver gauge theories, JHEP 11 (2009) 002 [arXiv:0907.2189] [INSPIRE].

[3] A. Mironov and A. Morozov, On AGT relation in the case of $\mathrm{U}(3)$, Nucl. Phys. B 825 (2010) 1 [arXiv: 0908.2569] [INSPIRE].

[4] L.F. Alday and Y. Tachikawa, Affine SL(2) conformal blocks from 4d gauge theories, Lett. Math. Phys. 94 (2010) 87 [arXiv:1005.4469] [INSPIRE].

[5] M.-C. Tan, M-Theoretic Derivations of 4d-2d Dualities: From a Geometric Langlands Duality for Surfaces, to the AGT Correspondence, to Integrable Systems, JHEP 07 (2013) 171 [arXiv:1301.1977] [INSPIRE].

[6] A. Belavin, V. Belavin and M. Bershtein, Instantons and 2d Superconformal field theory, JHEP 09 (2011) 117 [arXiv:1106.4001] [INSPIRE]. 
[7] V. Belavin and N. Wyllard, $N=2$ superconformal blocks and instanton partition functions, JHEP 06 (2012) 173 [arXiv: 1205.3091] [INSPIRE].

[8] V. Belavin and B. Feigin, Super Liouville conformal blocks from $N=2 \mathrm{SU}(2)$ quiver gauge theories, JHEP 07 (2011) 079 [arXiv: 1105.5800] [INSPIRE].

[9] M. Alfimov and G. Tarnopolsky, Parafermionic Liouville field theory and instantons on ALE spaces, JHEP 02 (2012) 036 [arXiv: 1110.5628] [INSPIRE].

[10] Y. Ito, Ramond sector of super Liouville theory from instantons on an ALE space, Nucl. Phys. B 861 (2012) 387 [arXiv:1110.2176] [InSPIRE].

[11] A. Belavin and B. Mukhametzhanov, $N=1$ superconformal blocks with Ramond fields from AGT correspondence, JHEP 01 (2013) 178 [arXiv: 1210.7454] [INSPIRE].

[12] N. Wyllard, Coset conformal blocks and $N=2$ gauge theories, arXiv:1109.4264 [INSPIRE].

[13] N. Nekrasov and A. Okounkov, Seiberg-Witten theory and random partitions, hep-th/0306238 [INSPIRE].

[14] R. Flume and R. Poghossian, An algorithm for the microscopic evaluation of the coefficients of the Seiberg-Witten prepotential, Int. J. Mod. Phys. A 18 (2003) 2541 [hep-th/0208176] [INSPIRE].

[15] H. Nakajima, Heisenberg algebra and Hilbert schemes of points on projective surfaces, Ann. Math. 145 (1997) 379. [alg-geom/9507012].

[16] H. Nakajima, Quiver varieties and finite dimensional representations of quantum affine algebras, Duke Math. J. 91 (1998) 515 [math/9912158].

[17] M. Atiyah and R. Bott, The moment map and equivariant cohomology, Topology 23 (1984) 1 [INSPIRE].

[18] A. Belavin, M. Bershtein, B. Feigin, A. Litvinov and G. Tarnopolsky, Instanton moduli spaces and bases in coset conformal field theory, Comm. Math. Phys. 319 1, pp 269-301 (2013) 269 [arXiv:1111.2803] [INSPIRE].

[19] V.A. Alba, V.A. Fateev, A.V. Litvinov and G.M. Tarnopolskiy, On combinatorial expansion of the conformal blocks arising from AGT conjecture, Lett. Math. Phys. 98 (2011) 33 [arXiv: 1012.1312] [INSPIRE].

[20] A. Belavin, M. Bershtein and G. Tarnopolsky, Bases in coset conformal field theory from AGT correspondence and Macdonald polynomials at the roots of unity, JHEP 03 (2013) 019 [arXiv:1211.2788] [INSPIRE].

[21] S. Fujii and S. Minabe, A Combinatorial Study on Quiver Varieties, ArXiv Mathematics e-prints October (2005). [math/0510455] [INSPIRE].

[22] P. Goddard, A. Kent and D.I. Olive, Virasoro Algebras and Coset Space Models, Phys. Lett. B 152 (1985) 88 [InSPIRE].

[23] C. Crnkovic, R. Paunov, G. Sotkov and M. Stanishkov, Fusions of conformal models, Nucl. Phys. B 336 (1990) 637 [inSPIRE].

[24] L. Spodyneiko, Implicit symmetries of the composite models of conformal field theory, unpublished.

[25] M.Y. Lashkevich, Superconformal $2-D$ minimal models and an unusual coset construction, Mod. Phys. Lett. A 8 (1993) 851 [hep-th/9301093] [InSPIRE]. 
[26] S.L. Lukyanov and V. Fateev, Exactly solvable models of conformal quantum theory associated with simple lie algebra D(n), (in Russian), Sov. J. Nucl. Phys. 49 (1989) 925 [INSPIRE].

[27] P. Goddard, A. Kent and D.I. Olive, Unitary Representations of the Virasoro and Supervirasoro Algebras, Commun. Math. Phys. 103 (1986) 105 [INSPIRE].

[28] P. Baseilhac and V. Fateev, Fermion boson duality in integrable quantum field theory, Mod. Phys. Lett. A 13 (1998) 2807 [hep-th/9905221] [INSPIRE].

[29] M. Bershtein, V. Fateev and A. Litvinov, Parafermionic polynomials, Selberg integrals and three-point correlation function in parafermionic Liouville field theory,

Nucl. Phys. B 847 (2011) 413 [arXiv:1011.4090] [INSPIRE].

[30] Z. Kakushadze and S.H. Tye, Kac and new determinants for fractional superconformal algebras, Phys. Rev. D 49 (1994) 4122 [hep-th/9310160] [INSPIRE].

[31] A. Belavin, A.M. Polyakov and A. Zamolodchikov, Infinite Conformal Symmetry in Two-Dimensional Quantum Field Theory, Nucl. Phys. B 241 (1984) 333 [inSPIRE].

[32] A. Belavin and D. Gepner, Generalized Rogers Ramanujan Identities from AGT Correspondence, Lett. Math. Phys. (2013) [arXiv:1212.6600] [INSPIRE].

[33] G. Bonelli, K. Maruyoshi and A. Tanzini, Gauge Theories on ALE Space and Super Liouville Correlation Functions, Lett. Math. Phys. 101 (2012) 103 [arXiv:1107.4609] [INSPIRE].

[34] G. Bonelli, K. Maruyoshi, A. Tanzini and F. Yagi, $N=2$ gauge theories on toric singularities, blow-up formulae and W-algebrae, JHEP 01 (2013) 014 [arXiv:1208.0790] [INSPIRE].

[35] N.A. Nekrasov, Seiberg-Witten prepotential from instanton counting, Adv. Theor. Math. Phys. 7 (2004) 831 [hep-th/0206161] [INSPIRE].

[36] F. Fucito, J.F. Morales and R. Poghossian, Multi instanton calculus on ALE spaces, Nucl. Phys. B 703 (2004) 518 [hep-th/0406243] [INSPIRE].

[37] F. Fucito, J.F. Morales and R. Poghossian, Instanton on toric singularities and black hole countings, JHEP 12 (2006) 073 [hep-th/0610154] [INSPIRE].

[38] Y. Ito, K. Maruyoshi and T. Okuda, Scheme dependence of instanton counting in ALE spaces, JHEP 05 (2013) 045 [arXiv: 1303.5765] [INSPIRE].

[39] D. Gaiotto, Asymptotically free $N=2$ theories and irregular conformal blocks, arXiv:0908.0307 [INSPIRE].

[40] A. Marshakov, A. Mironov and A. Morozov, On non-conformal limit of the AGT relations, Phys. Lett. B 682 (2009) 125 [arXiv: 0909. 2052] [INSPIRE].

[41] V. Fateev and S.L. Lukyanov, The Models of Two-Dimensional Conformal Quantum Field Theory with $Z_{n}$ Symmetry, Int. J. Mod. Phys. A 3 (1988) 507 [INSPIRE]. 